\title{
Effect of Cultivation Methods on Seed Yield Traits, Oil Yield, and Fatty Acid Compositions of Cultivated Paeonia
}

\section{ostii}

\author{
Tong Zhang, Zheng Zhang, Qi Qiao, Wei Liu, and Xiaogai Hou \\ College of Agriculture, Henan University of Science and Technology, Luoyang \\ 471000, China
}

Additional index words. $\alpha$-linolenic acid, cultivation, oil-use feature, seed oil, tree peony

\begin{abstract}
Paeonia ostii is recognized as an important oilseed tree peony species with potential as a raw material source for cosmetic and health care products, strong seed setting capacity, high seed oil yield, and abundant omega-3 polyunsaturated fatty acids. $P$. ostii, commonly called Fengdan, is widely cultivated in China. The cultivation method difference has an important influence on the oil-use feature, which is a key index for evaluating the quality of oilseed crops. This study aimed to select an optimal cultivation method to provide the first reference data for high-yield and high-quality seed oil harvesting and to facilitate the understanding of the quality difference in the formation mechanism of seed oil in cultivated $P$. ostii. This study selected five representative cultivation methods, open field cultivation, sunny slope cultivation, shady slope cultivation, understory intercropping cultivation, and high-altitude cultivation, and investigated the influence of cultivation method differences on the oil-use feature based on the three aspects of seed yield traits, oil yield, and fatty acid compositions. Six seed yield traits (fruit pod length, fruit pod width, fruit pod thickness, number of fruit pods per plant, 1000-grain weight, and seed yield per plant) and oil yield reached the maximum values of $7.75 \mathrm{~cm}, 6.99 \mathrm{~cm}, 1.57 \mathrm{~cm}, 11.33,290.45 \mathrm{~g}, 85.8 \mathrm{~g}$, and $30.41 \%$, respectively, using the understory intercropping cultivation method. Fatty acid compositions were mainly identified as $\alpha$-linolenic acid, linoleic acid, oleic acid, stearic acid, and palmitic acid, with significant content differences among different cultivation methods $(P<0.05)$. Functional component $\alpha$-linolenic acid and total amounts of unsaturated fatty acids reached the maximum values of $46.85 \%$ and $65.23 \%$, respectively, with high-altitude cultivation and understory intercropping cultivation. The seed yield traits, oil yield, and total amounts of unsaturated fatty acids were optimal with the understory intercropping cultivation method, whereas high-altitude cultivation was conducive to the accumulation of $\alpha$-linolenic acid. Understory intercropping cultivation with appropriate altitude increases is recommended as a preponderant cultivation method for high-yield and high-quality seed oil harvesting for this crop.
\end{abstract}

$\alpha$-Linolenic acid (ALA) is rich in seed oil of tree peony. As an innovative and emerging woody oilseed crop resource, tree peony is now being more effectively developed and used in China. Paeonia ostii belongs to the perennial deciduous shrub of Sect. Moutan DC. of Paeonia in Paeoniaceae (Flora of China Editorial Committee, 2001). There are nine wild species of Paeonia Sect. Moutan according to Li's taxonomy system $(\mathrm{Li}, 2011)$ ( $P$. suffruticosa, $P$. ostii, $P$. rockii, $P$. delavayi, $P$. cathayana, $P$. ludlowii, $P$. decomposita, $P$.

Received for publication 18 Aug. 2021. Accepted for publication 10 Sept. 2021

Published online 27 October 2021

This work was supported by the Program for Zhongyuan Scholars of Henan Province (No. 212101510003) and the Key Scientific Research Projects of the Higher Education Institutions of Henan Province (No. 19A180002). We give special thanks to other colleagues in the same laboratory for helpful comments regarding the manuscript.

W.L. and X.H. are the corresponding authors. E-mail: 15729111052@163.com or hkdhxg@126.com.

This is an open access article distributed under the CC BY-NC-ND license (https://creativecommons. org/licenses/by-nc-nd/4.0/). jishanensis, and $P$. qiui). Tree peony is native to China and an important horticultural plant with great ornamental value. It is also a traditional famous flower and commonly known as the "king of the flowers" because it represents wealth and glory $(\mathrm{Li}, 2011)$. In addition, the root cortex of tree peony is commonly applied as traditional Chinese medicine (TCM) material called Moutan cortex that has analgesic, sedative, and anti-inflammatory functions because of high paeonol and paeonoside produced in its root cortex (Lin et al., 2015; Zhang et al., 2017a, 2018a). As a special and precious flower in China, $P$. ostii was found to have high oil-use value in addition to ornamental and medicinal applications. In Mar. 2011, seed oils from $P$. ostii and $P$. rockii were approved as new resource foods among the nine wild species of tree peony by the Food and Drug Administration of China (Shi et al., 2014). Currently, as an important oil-use tree peony variety, $P$. ostii is cultivated on a large-scale for oil extraction, which gradually forms a single and stable tree peony cultivar and is commonly known as Fengdan in Chinese.

The oil-use value of tree peony was initially found in 1970, and has been given more attention since 2004 because tree peony seeds were found to contain ALA, linoleic acid, peony sterol, and other active substances (Wei et al., 2018). P. ostii seed oil contains various fatty acid components and unsaponifiable compounds, with the unsaturated fatty acid content exceeding $90 \%$. As a functional component of seed oil, ALA accounts for nearly half of the total fatty acids (Han et al., 2014). The ALA contents and unsaturated fatty acids contents in $P$. ostii seed oil are much higher than those in the main edible vegetable oils circulating on the market. For example, in rapeseed oil, soybean oil, olive oil, and peanut oil, the ALA content was less than $10 \%$, and the unsaturated fatty acids contents only accounted for $\approx 80 \%$ (de Andrade et al., 2019; Konuskan and Mungan, 2016, 2019; Mariela et al., 2017). The unsaturated fatty acids are rich in $P$. ostii seed oil, including ALA, oleic acid, and linoleic acid, which are necessary for the human body. These compounds exhibit anti-cardiovascular disease, anti-inflammatory, anti-cancer, and hypoglycemic effects, and they promote brain development (Wang et al., 2016; Zhang et al., 2016). In addition, seed oil of $P$. ostii have strong antioxidant activity and sunscreen effects (Gao et al., 2013; Zhang et al., 2017a, 2017b; Zhang et al., 2018a). It is well-known that omega-3 fatty acids help in the treatment of and defense against cardiovascular and cerebrovascular diseases, stroke, and rheumatoid arthritis. However, natural omega-3 fatty acids sources are limited; they are only found in deep-sea fishes, seal oil, and some special plant species (Nichols et al., 2010). Therefore, $P$. ostii is a new plant resource for natural sources of omega-3 fatty acids. As an alternative source of omega- 3 fatty acids, $P$. ostii has different uses in many fields, such as the health care, food, cosmetics, and pharmaceutical industries (Han et al., 2016).

Although seed oil of $P$. ostii has entered the market as edible oil, its seed yield is very low under the current planting pattern. Consequently, high-yield and high-quality largescale planting of $P$. ostii has not yet occurred. Seed yield traits, oil yield, and fatty acid compositions are important indexes to evaluate oilseed crop quality, but these indexes are restricted by many factors, such as cultivation methods, environmental factors, and genetic factors during the planting period. At present, few studies have focused on the effects of different cultivation methods on the seed yield traits, oil yield, and fatty acid compositions of cultivated $P$. ostii. Only the preliminary studies performed by Qin (2016) and Song et al. (2018) are available. Therefore, in the present study, five representative cultivation methods were selected to assess the response of $P$. ostii to different cultivation methods from the three aspects of seed yield traits, seed oil yield, and seed oil fatty acid compositions. Furthermore, the preponderant cultivation method was screened and suggested based on five test cultivation methods using integrative comparison and data analysis. This work provides a new theoretical basis for the high-yield and high-quality cultivation 
of $P$. ostii and facilitates the understanding of the mechanism of quality differences in $P$. ostii and other oilseed crops. These results may promote the sustainable development and application extension of the oilseed tree peony industry.

\section{Materials and Methods}

Main reagents and instruments. During this study, n-hexane (analytically pure) was purchased from Tianjin Damao Chemical Reagent Factory (China). Methanol (analytically pure) was purchased from Tianjin Fengchuan Chemical Reagent Technology Co., Ltd. (China) and $98.08 \%$ concentrated sulfuric acid (analytically pure) was purchased from Haohua Chemical Reagent Co., Ltd. (Luoyang City, China). Sodium hydroxide (analytically pure) and anhydrous sodium sulfate (analytically pure) were purchased from Tianjin Kaitong Chemical Reagent Co., Ltd. (China). Sodium chloride (analytically pure) was purchased from Tianjin Fenghua Chemical Reagent Factory, China. Anhydrous ethanol (analytically pure) was purchased from Tianjin Beichen Fangzheng Reagent Factory (China). Tridecanoic acid methylester standard (analytically pure) was purchased from NU-CHEK (Elysian, MN).

The vernier caliper was purchased from Suzhou Jingyi Precision Instrument Co., Ltd. (China). The BSA124S analytical balance was purchased from GL International Trade (Shanghai) Co., Ltd. (China). The T-400B highspeed multifunction pulverizer was purchased from Dingshuai Hardware Products Co., Ltd. (Yongkang City, China). The serpentine Soxhlet extractor was purchased from Shanghai Leigu Instrument Co., Ltd. (China). The R$1001 \mathrm{VN}$ rotary evaporator was purchased from Changcheng Science and Industry Co., Ltd. (Zhengzhou City, China). The DSQ II gas chromatography-mass spectrometer was purchased from Thermo Fisher Scientific (Waltham, MA).

Plant materials. The experiment area is located in the Oilseed Tree Peony Test Base of Alpine Tree Peony Garden in Luoling Town of Luoyang City in China (lat. $34^{\circ} 22^{\prime} 18^{\prime \prime} \mathrm{N}$, long. $\left.111^{\circ} 20^{\prime} 7^{\prime \prime} \mathrm{E}\right)$. Five different cultivation types of $P$. ostii can be performed in this experimental area, namely, traditional open field cultivation $(150 \mathrm{~m})$, sunny slope cultivation (150 m), shady slope cultivation $(150 \mathrm{~m})$, understory intercropping $(150 \mathrm{~m})$, and high-altitude cultivation (810 $\mathrm{m})$. Some artificial measures were performed to remove weeds under the plants. Timely and appropriate watering was applied, followed by deep turning in March and May each year. No other field management measures were used except for these normal management procedures. The test area is located in the temperate continental monsoon climate zone. The average maximum temperature in summer is $33.2^{\circ} \mathrm{C}$. The average minimum temperature in winter is $-4.3^{\circ} \mathrm{C}$. The average annual temperature is $13.7^{\circ} \mathrm{C}$. The average annual precipitation is $613.6 \mathrm{~mm}$. The average annual sunshine hours are $2450.35 \mathrm{~h}$. The four seasons are distinctive, with a frost-free period of $216 \mathrm{~d}$. The soil type is gravel loam.

Plant spacing was $0.5 \mathrm{~m} \times 0.6 \mathrm{~m}$. The understory intercropping cultivation method involved $P$. ostii planted under the Ginkgo biloba forest. The ginkgo tree was 8 years old, with a tree height of 2 to $4 \mathrm{~m}$ and a canopy density of 0.2 to 0.45 . Particularly, the altitude of the high-altitude cultivation method was $810 \mathrm{~m}$. Using the five cultivation methods, plant materials were collected from $3-\times 5-\mathrm{m}$ $\times 5$-m sample plots that had been planted for 6 years during 18 Aug. 2019. The samples were identified as the oilseed tree peony $P$. ostii cultivars by Professor Xue Xian of Henan University of Science and Technology.
Measurement of key traits of seed yield in $P$. ostii. Seed yield traits were determined after the ripening of $P$. ostii seeds. The mature seeds were collected when the seed became brown (Fig. 1) (Xue et al., 2015). The six key seed yield traits for determination included the fruit pod length, fruit pod width, fruit pod thickness, number of fruit pods per plant, 1000-grain weight, and seed yield per plant. For plant individuals of $P$. ostii from each cultivation type, the fruit pod length, fruit pod width, and fruit pod thickness were measured with a vernier caliper, and the measurement data were accurate to $0.01 \mathrm{~cm}$. All fruit pods were collected and then stored according to the sample mark. After indoor natural drying, the number of fruit pods was counted. An electronic balance was used to measure the 1000-grain weight and seed yield per plant, and the measurement data were accurate to $0.01 \mathrm{~g}$.

Extraction of seed oil. The seed oil was extracted using the Soxhlet extraction method as previously described by Bai et al. (2017). $P$. ostii seeds were ground into fine powder, dried at $50{ }^{\circ} \mathrm{C}$ for $3 \mathrm{~h}$ in the drying oven, cooled to room temperature, and then filtered with a 40 mesh sieve. Then, $10 \mathrm{~g}$ of $P$. ostii seed powder was enclosed in a stacked filter paper bag and placed in the extraction tube. A $100-\mathrm{mL}$ aliquot of $n$-hexane was placed in a round-bottom flask. The heater (electronic temperature heating jacket) was first set to $80^{\circ} \mathrm{C}$ to boil n-hexane, and then to $40^{\circ} \mathrm{C}$ for 3.5 -h extraction. The resulting product was concentrated and purified using a rotary evaporator, and the yellow translucent oily liquid in the rotary evaporation bottle was transferred to the brown reagent flask, which was marked separately and placed in a $-4.0^{\circ} \mathrm{C}$ refrigerator for storage. Under the same conditions, each sample underwent this procedure three times.

Oil yield calculation of $P$. ostii. The rotary evaporation flask $\left(\mathrm{m}_{1}\right)$ was weighed with an
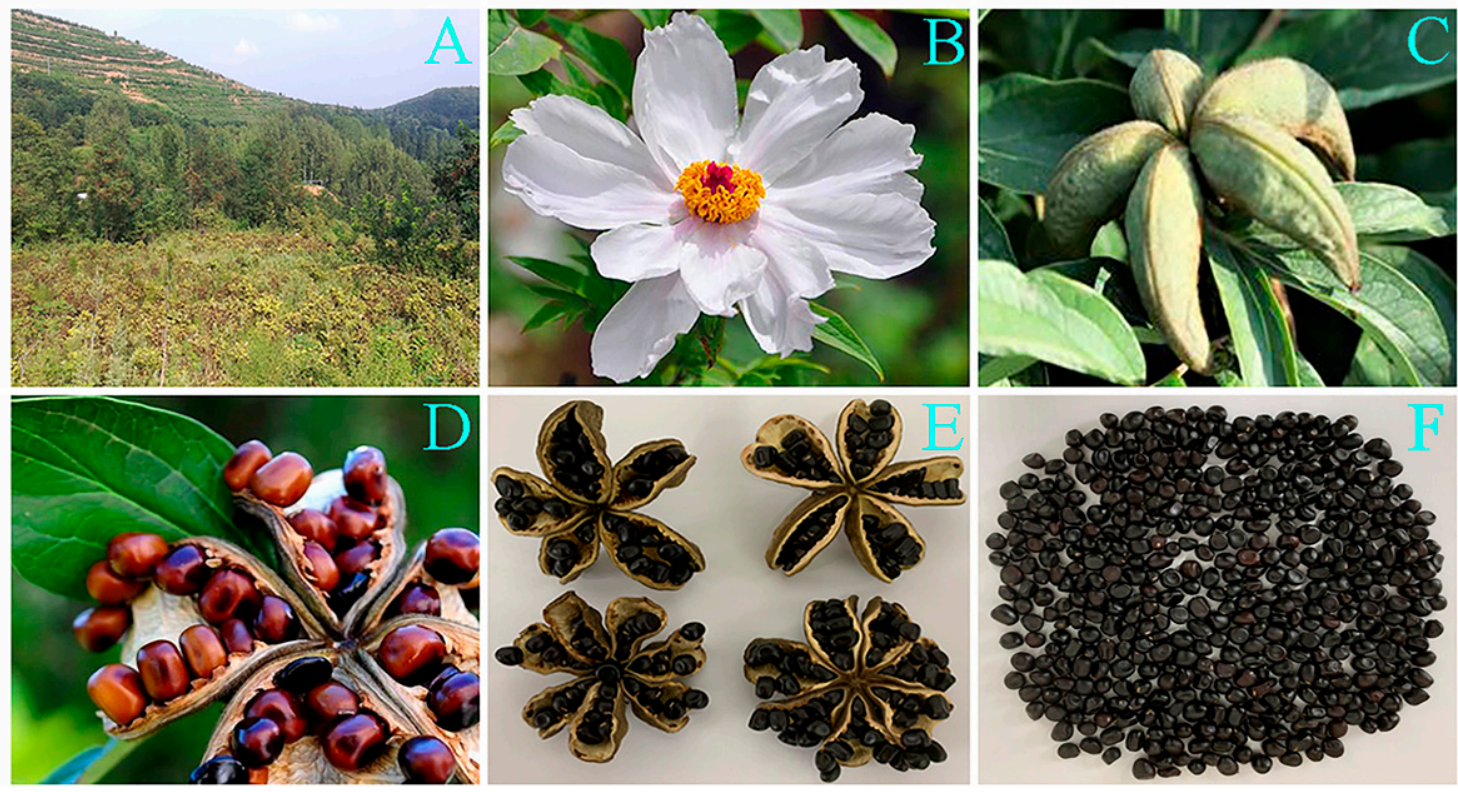

Fig. 1. Blooming and seed setting of Paeonia ostii. (A) Oilseed tree peony test base. (B) Flower of $P$. ostii. (C) Full fruit pod of $P$. ostii. (D) Fully mature fruit pods of $P$. ostii. (E) Dry mature fruit pod in the shade. (F) Dry mature seeds in the shade. 
electronic balance before each rotary evaporation. The rotary evaporation flask and the extraction products $\left(\mathrm{m}_{2}\right)$ were weighed after each rotary evaporation. Then, the oil yield was calculated for each sample according to the sample weight of $P$. ostii seed powder $\left(\mathrm{m}_{3}\right)$. The oil yield calculation formula was as follows:

$$
\text { Oil yield }=\left(m_{2}-m_{1}\right) / m_{3} \times 100 \%
$$

Quantification of the fatty acid composition in P. ostii seed oil. To prepare the internal standard solution, $10 \mathrm{mg}$ of the methyl tridecanoate standard was dissolved in $2.5 \mathrm{~mL}$ of n-hexane to form a standard solution with ae concentration of $4 \mathrm{mg} / \mathrm{mL}$. Then, it was placed into $\mathrm{a}-4.0^{\circ} \mathrm{C}$ refrigerator for storage.

To prepare the test sample solution, the methyl-esterification reaction was performed before the gas chromatography-mass spectrometry analysis of seed oil composition. This reaction was optimized and improved based on the methyl-esterification reaction method performed by Han et al. (2016). A $240-\mu \mathrm{L}$ aliquot of the seed oil was added in a stoppered test tube and mixed with $4 \mathrm{~mL}$ of $0.5 \mathrm{~mol} \cdot \mathrm{mL}^{-1} \mathrm{NaOH}-$ methanol solution. The mixture was subjected to a water bath with a constant temperature of $60^{\circ} \mathrm{C}$ after fully blending; then, it was shaken once every 5 min until the seed oil completely dissolved (this process requires $\approx 30 \mathrm{~min}$ ). Then, $2 \mathrm{~mL}$ of $5 \% \mathrm{H}_{2} \mathrm{SO}_{4}$-methanol solution was added to the tube after cooling to room temperature and subjected to a water bath for $10 \mathrm{~min}$ at a constant temperature of $60^{\circ} \mathrm{C}$. Then, $2 \mathrm{~mL}$ of $\mathrm{n}$-hexane was added to the tube after cooling to room temperature; it was mixed with $2 \mathrm{~mL}$ of saturated $\mathrm{NaCL}$ solution, which was allowed to stand for stratification. The upper liquid was collected in a centrifuge tube, and an appropriate amount of anhydrous sodium sulfate was added for dehydration. The upper liquid was collected with a 2-mL syringe after centrifugation and filtered through a $0.22-\mu \mathrm{m}$ organic microfiltration membrane as the test sample solution. Then, $0.9 \mathrm{~mL}$ of test sample solution and $0.1 \mathrm{~mL}$ of internal standard solution were added to a sample flask for further gas chromatography-mass spectrometry analyses.

Gas chromatography-mass spectrometry analysis of fatty acid composition of $P$. ostii seed oil. The gas chromatography-mass spectrometry method was used to analyze the fatty acid composition of $P$. ostii seed oil. The column was a TG-5MS $(30.0 \mathrm{~m} \times 0.25$ $\mathrm{mm} ; 0.25 \mu \mathrm{m})$ elastic quartz capillary column. The carrier gas was high-purity helium
$(99.999 \%)$ with a flow rate of $5 \mathrm{~mL} / \mathrm{min}$. The injection mode was an 80:1 shunting sample injection with the injection volume of $2 \mu \mathrm{L}$, and the injection temperature was $260^{\circ} \mathrm{C}$. The temperature of the setup program was $80^{\circ} \mathrm{C}$, which was maintained for $1 \mathrm{~min}$ and then increased to $180^{\circ} \mathrm{C}$ at a rate of $40^{\circ} \mathrm{C} /$ min for $1 \mathrm{~min}$, to $190^{\circ} \mathrm{C}$ at a rate of $1{ }^{\circ} \mathrm{C} / \mathrm{min}$ for $5 \mathrm{~min}$, and finally to $205^{\circ} \mathrm{C}$ at a rate of $1{ }^{\circ} \mathrm{C} / \mathrm{min}$ for $5 \mathrm{~min}$. The column flow rate was $5.0 \mathrm{~mL} / \mathrm{min}$, and the gas chromatography-mass spectrometry interface temperature was $250^{\circ} \mathrm{C}$. The ion source was EI, and the ion source temperature was $250^{\circ} \mathrm{C}$. The temperature of the transmission line was $250^{\circ} \mathrm{C}$. The power voltage was $70 \mathrm{eV}$, and the scan range $(\mathrm{m} / \mathrm{z})$ was 30 to $460 \mathrm{amu}$, with a scan time of $0.2 \mathrm{~s}$.

Measurement of type and content of fatty acid compositions. The retention time of the chromatographic peak was compared between the fatty acid methylester standard and the test samples. The NIST2011 spectrum library was used to determine the type of fatty acid composition in each sample based on the ion quality of the chromatographic peak in the resulting spectra. During a comparison of the chromatography peak area of the standard methyl tridecanoate and test samples, each fatty acid composition content was calculated using an internal standard method.

Statistical analysis. All data were calculated by Excel 2016 (Microsoft Corporation, Redmond, WA) and represented as mean \pm SD $(n=3)$. Origin 9.0 (OriginLab Corporation, Northampton, MA) was used for related graph creation. SPSS 25.0 software (IBM SPSS Inc., Chicago, IL) was used for the one-way analysis of variance and Duncan's multiple comparisons $(P<0.05)$.

\section{Results}

Difference in the seed yield traits of $P$. ostii using different cultivation methods. During this study, six key agronomic traits (fruit pod length, fruit pod width, fruit pod thickness, number of fruit pods per plant, 1000grain weight, and seed yield per plant) were selected to examine the effects of different cultivation methods on the seed yield of $P$. ostii (Table 1). These seed yield traits reached their maximum values with the understory intercropping cultivation method. There were no significant differences in the fruit pod length found with the understory intercropping cultivation method $(7.75 \mathrm{~cm})$ and highaltitude cultivation $(7.01 \mathrm{~cm})$, but the former value was significantly higher than that with the sunny slope cultivation method (6.59 $\mathrm{cm})$, the shady slope cultivation method (6.19 $\mathrm{cm})$, and the open field cultivation method $(6.44 \mathrm{~cm})(P<0.05)$. The fruit pod width was significantly higher with the understory intercropping cultivation method $(6.99 \mathrm{~cm})$ than that with the shady slope cultivation method $(5.65 \mathrm{~cm})$, but no significant difference was found compared with other cultivation methods $(P<0.05)$. There were no significant differences in fruit pod thickness between the understory intercropping cultivation method $(1.57 \mathrm{~cm})$ and the high-altitude cultivation method $(1.48 \mathrm{~cm})$, but the former value was significantly higher than that with the sunny slope $(1.35 \mathrm{~cm})$, the shady slope $(1.36 \mathrm{~cm})$, and the open field cultivation methods $(1.37 \mathrm{~cm})(P<0.05)$. A significant difference in the number of fruit pods per plant was not found among all the cultivation methods $(P<0.05)$, indicating that the number of fruit pods per plant was not restricted by the cultivation method, or that the different cultivation methods had no significant effect on the number of fruit pods per plant. The 1000-grain weight is an important index for testing and predicting the yield of seeds in the field, and its value can reflect the seed quality and seed fullness (Duan et al., 2018; Zhang et al., 2018b). The 1000-grain weight with the understory intercropping cultivation method (290.45 g) had no significant difference compared with the high-altitude cultivation method (277.93 g), but the former value was significantly higher than that with the sunny slope $(251.99 \mathrm{~g})$, the shady slope $(239.95 \mathrm{~g})$, and the open field cultivation methods $(253.89 \mathrm{~g})(P<0.05)$. No significant difference in seed yield per plant was observed among the understory intercropping cultivation method $(85.80 \mathrm{~g})$, high-altitude cultivation method $(77.95 \mathrm{~g})$, and shady slope cultivation method (78.45 g), but the former value was significantly higher than that with the sunny slope $(64.83 \mathrm{~g})$ and open field cultivation methods $(54.98 \mathrm{~g})$, respectively $(P<$ $0.05)$. These results indicated that all seed yield traits reached their optimal values with the understory intercropping cultivation method, followed by the high-altitude cultivation method, and no significant differences were found between both methods $(P<0.05)$.

Difference in the oil yield of $P$. ostii under different cultivation methods. Seed oil yield is a key indicator for evaluating the oil-use features of oilseed crops (Luo et al., 2016). The effects of different cultivation methods on the

Table 1. Differentiation of the seed yield traits of Paeonia ostii with different cultivation methods.

\begin{tabular}{|c|c|c|c|c|c|c|}
\hline Cultivation methods & $\begin{array}{c}\text { Fruit pod } \\
\text { length }(\mathrm{cm})\end{array}$ & $\begin{array}{c}\text { Fruit pod } \\
\text { width }(\mathrm{cm})\end{array}$ & $\begin{array}{l}\text { Fruit pod } \\
\text { thickness }(\mathrm{cm})\end{array}$ & $\begin{array}{l}\text { Number of fruit } \\
\text { pods per plant }\end{array}$ & $\begin{array}{l}\text { 1000-grain } \\
\text { wt (g) }\end{array}$ & $\begin{array}{c}\text { Seed yield } \\
\text { per plant }(g)\end{array}$ \\
\hline Sunny slope cultivation & $6.59 \pm 0.80 \mathrm{~b}$ & $6.18 \pm 0.82 \mathrm{ab}$ & $1.35 \pm 0.05 \mathrm{~b}$ & $10.00 \pm 1.00 \mathrm{a}$ & $251.99 \pm 10.32 \mathrm{~b}$ & $64.83 \pm 6.03 \mathrm{~b}$ \\
\hline Shady slope cultivation & $6.19 \pm 0.24 b$ & $5.65 \pm 0.20 \mathrm{~b}$ & $1.36 \pm 0.06 \mathrm{~b}$ & $9.67 \pm 0.58 \mathrm{a}$ & $239.95 \pm 1.57 \mathrm{~b}$ & $78.45 \pm 5.84 \mathrm{a}$ \\
\hline High-altitude cultivation & $7.01 \pm 0.44 \mathrm{ab}$ & $6.53 \pm 0.36 \mathrm{ab}$ & $1.48 \pm 0.13 \mathrm{ab}$ & $10.67 \pm 1.15 \mathrm{a}$ & $277.93 \pm 14.91 \mathrm{a}$ & $77.95 \pm 4.43 \mathrm{a}$ \\
\hline $\begin{array}{l}\text { Understory intercropping } \\
\text { cultivation }\end{array}$ & $7.75 \pm 0.31 \mathrm{a}$ & $6.99 \pm 0.27 \mathrm{a}$ & $1.57 \pm 0.07 \mathrm{a}$ & $11.33 \pm 0.58 \mathrm{a}$ & $290.45 \pm 2.55 \mathrm{a}$ & $85.80 \pm 2.66 \mathrm{a}$ \\
\hline Open field cultivation & $6.44 \pm 0.71 b$ & $5.94 \pm 0.83 \mathrm{ab}$ & $1.37 \pm 0.07 \mathrm{~b}$ & $10.00 \pm 1.00 \mathrm{a}$ & $253.89 \pm 13.1 \mathrm{~b}$ & $54.98 \pm 4.65 \mathrm{c}$ \\
\hline
\end{tabular}

Different lowercase letters in the same column represent significant differences among different treatments $(P<0.05)$. 
Table 2. One-way analysis of variance and Duncan multiple comparisons for oil yield of Paeonia ostii with different cultivation methods.

\begin{tabular}{|c|c|c|c|c|c|c|c|}
\hline Cultivation methods & Avg oil yield (\%) & SD & $\begin{array}{l}\text { Variation } \\
\text { coefficient }\end{array}$ & $\begin{array}{l}\text { Maximum } \\
\text { value (\%) }\end{array}$ & $\begin{array}{l}\text { Minimum } \\
\text { value (\%) }\end{array}$ & Range test & $P$ value \\
\hline Sunny slope cultivation & 28.94 & 2.74 & 9.73 & 31.42 & 27.00 & 4.42 & 0.0735 \\
\hline Shady slope cultivation & 29.33 & 3.26 & 11.12 & 32.99 & 26.73 & 6.26 & 0.0857 \\
\hline Understory intercropping cultivation & 30.41 & 1.70 & 5.71 & 32.18 & 28.18 & 4.00 & 0.0211 \\
\hline Open field cultivation & 28.18 & 3.13 & 10.27 & 32.00 & 25.63 & 6.37 & 0.2394 \\
\hline
\end{tabular}

oil yield of $P$. ostii were investigated during this study (Table 2). Using different cultivation methods, the ranking order of oil yield was as follows: understory intercropping cultivation $(30.41 \%)>$ high-altitude cultivation $(29.51 \%)$ $>$ shady slope cultivation $(29.33 \%)>$ sunny slope cultivation $(28.94 \%)>$ open field cultivation $(28.18 \%)$. The oil yield of $P$. ostii reached its maximum with the understory intercropping cultivation method, followed by the high-altitude cultivation method, which had a significantly higher value than the values reached with other cultivation methods $(P<0.05)$.

The lowest variation coefficient of oil yield (5.71) was found for the understory intercropping cultivation method, indicating that the oil yield is relatively stable with this cultivation method. Compared with the other three cultivation methods (sunny slope cultivation, shady slope cultivation, and open field cultivation), the oil yield with the high-altitude cultivation method was also relatively stable, with a small variation coefficient of 6.34. The average oil yield of $P$. ostii seeds was $28.18 \%$ to $30.41 \%$, which is consistent with the results described by Han et al. (2016), Luo et al. (2016) and Zhang et al. (2017c). The highest and most stable oil yield of $P$. ostii was observed with the understory intercropping cultivation method, followed by the high-altitude cultivation method. Therefore, the understory intercropping cultivation method is conducive to the seed oil production of $P$. ostii.

Difference in the fatty acid composition in seed oil using different cultivation methods. The gas chromatography-mass spectrometry analysis results of fatty acids resulting from different cultivation methods are shown in
Figs. 2-6 and Figs. S1-S5. The contents of fatty acid compositions are presented in Table 3. As shown in Figs. 2-6 and Figs. S1-S5, seed oil of $P$. ostii included various fatty acid types. The five fatty acid types were unambiguously identified as ALA, linoleic acid, palmitic acid, stearic acid, and oleic acid, accounting for more than $55 \%$ of the total fatty acid content (Table 3 ). Therefore, these five substances were selected as the main representative ingredients to investigate the effects of different cultivation methods on the fatty acid compositions of $P$. ostii seed oil in this study.

Table 3 showed that the maximum content (46.85\%) of ALA was found with high-altitude cultivation and significantly different when compared with the other cultivation methods $(P<0.05)$. However, the minimum content of ALA $(37.19 \%)$ was obtained with the open field cultivation method. The maximum content of linoleic acid (20.08\%) was observed with the understory intercropping cultivation, which was significantly different from that with the other cultivation methods $(P<0.05)$, whereas the smallest content of linoleic acid (16.21\%) was obtained using the open cultivation method. In all the tested fatty acids, the oleic acid content was relatively low $(0.00233-0.0031 \%)$. The maximum content of oleic acid $(0.0031 \%)$ was found using shady slope cultivation, with indistinctive differences from the other cultivation methods $(P<0.05)$, followed by the sunny cultivation methods $(0.00294 \%)$. The minimum content $(0.00233 \%)$ was measured with the understory intercropping cultivation method.

The contents of palmitic acid reached the maximum with the high-altitude cultivation method (3.99\%) and the shady slope cultivation method $(3.98 \%)$, and there was no significant difference between them $(P<0.05)$. Palmitic acid reached the minimum value $(3.28 \%)$ with open field cultivation and was significantly lower than that with other cultivation methods $(P<0.05)$. The maximum $(1.29 \%)$ and minimum $(0.89 \%)$ contents of stearic acid were found with the shady slope cultivation method and open field cultivation method, respectively. The differences in the stearic acid contents among the five different cultivation methods were significant $(P<0.05)$. The total amounts of unsaturated fatty acids (ALA, linoleic acid, and oleic acid) reached the maximum value of $65.23 \%$ with the understory intercropping cultivation method and was significantly higher than those with the other four cultivation methods $(P<0.05)$. The content of saturated fatty acids (palmitic acid and stearic acid) reached the maximum value of $5.26 \%$ with the shady slope cultivation method and was significantly higher than those with the other four cultivation methods $(P<0.05)$. The results showed that the types of fatty acid compositions of $P$. ostii seed oil with different cultivation methods were nearly the same, but the contents of each composition displayed significant differences with the different cultivation methods $(P<$ $0.05)$. The highest content of ALA was obtained with the high-altitude cultivation. The highest linoleic acid contents and total unsaturated fatty acids contents were simultaneously found with the understory intercropping cultivation method. Therefore, high-altitude cultivation could produce abundant ALA, and understory intercropping cultivation could obtain better types and proportions of fatty acid compositions.

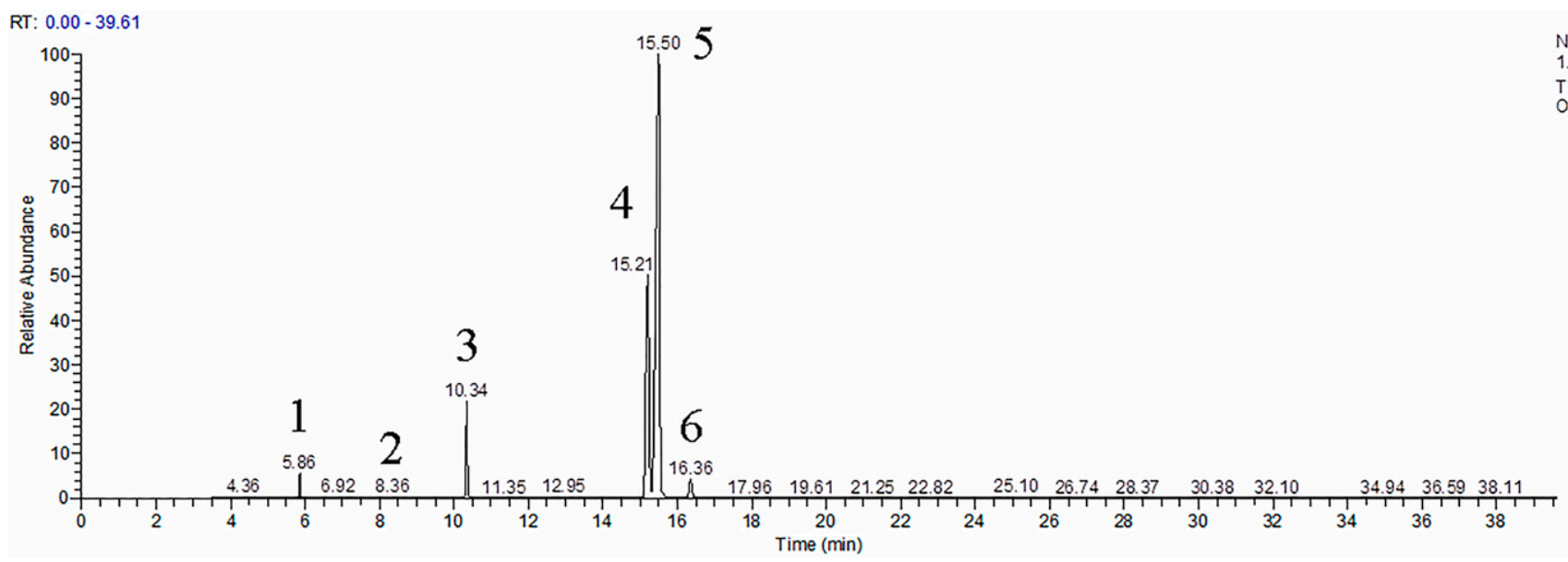

Fig. 2. Fatty acid methylester chromatogram for Paeonia ostii seed oil with the sunny slope cultivation method. Numbers 1 to 6 indicate tridecanoic acid, oleic acid, palmitic acid, linoleic acid, $\alpha$-linolenic acid, and stearic acid, respectively. 


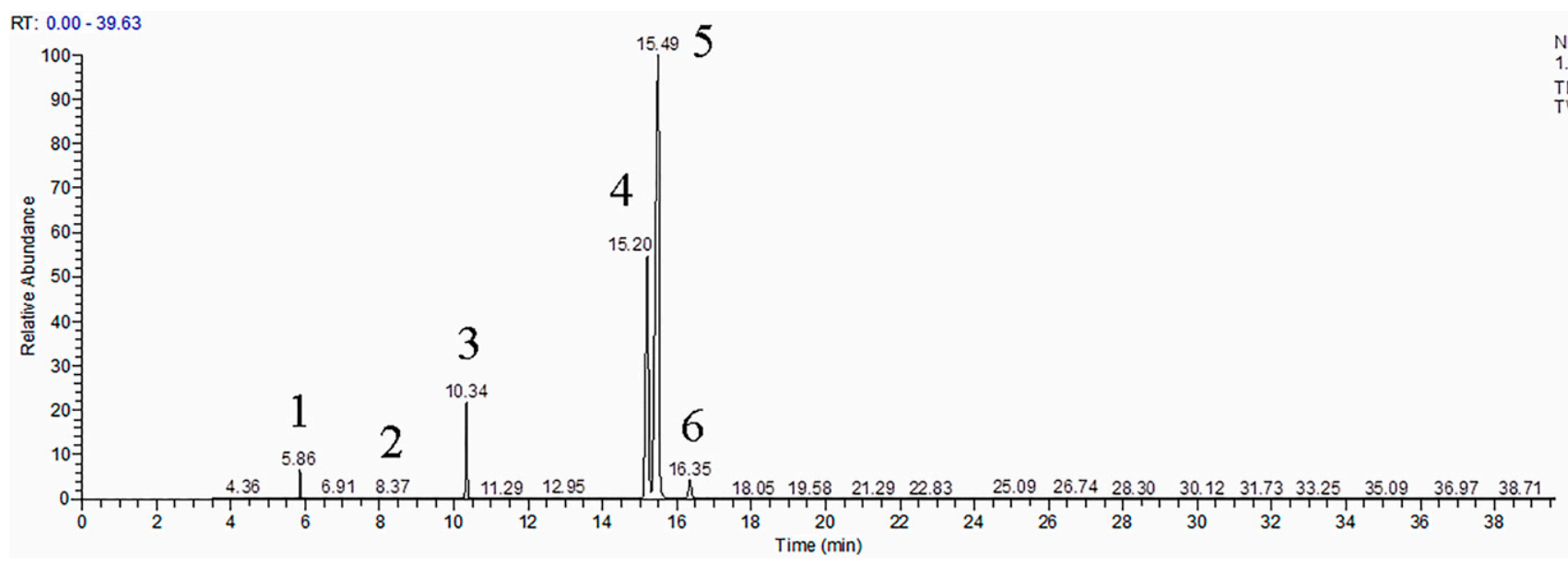

Fig. 3. Fatty acid methylester chromatogram for Paeonia ostii seed oil with the shady slope cultivation method. Numbers 1 to 6 indicate tridecanoic acid, oleic acid, palmitic acid, linoleic acid, $\alpha$-linolenic acid, and stearic acid, respectively.

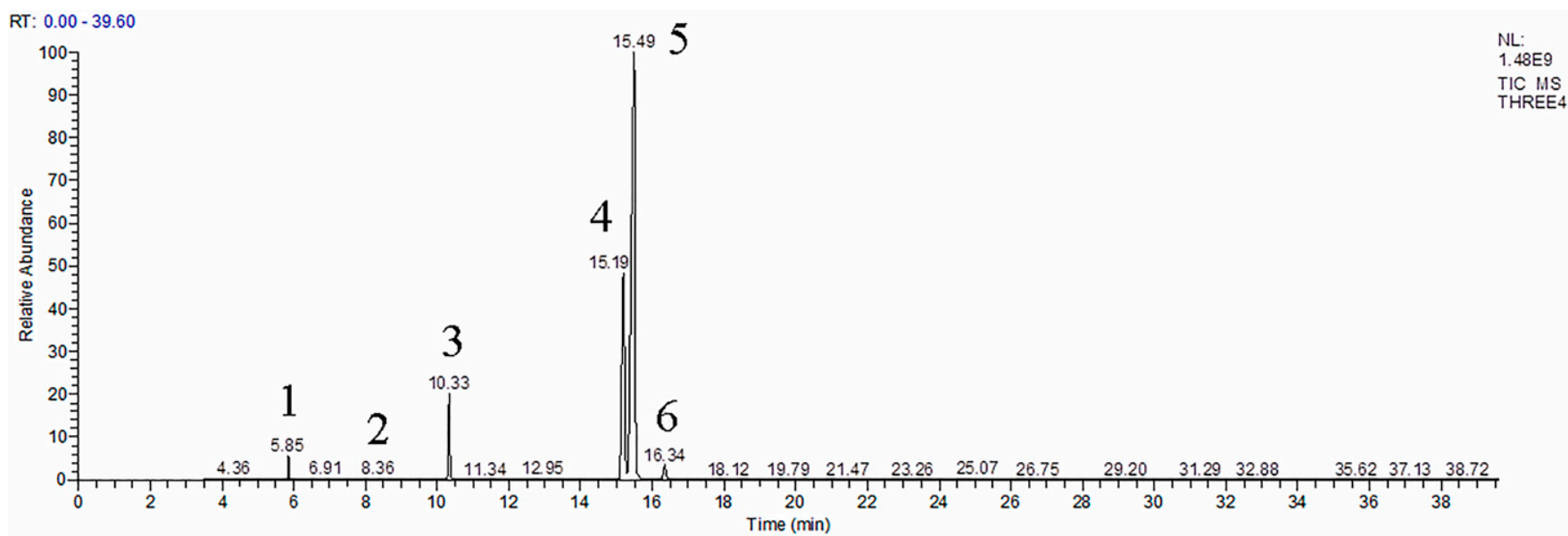

Fig. 4. Fatty acid methylester chromatogram for Paeonia ostii seed oil with the high-altitude cultivation method. Numbers 1 to 6 indicate tridecanoic acid, oleic acid, palmitic acid, linoleic acid, $\alpha$-linolenic acid, and stearic acid, respectively.

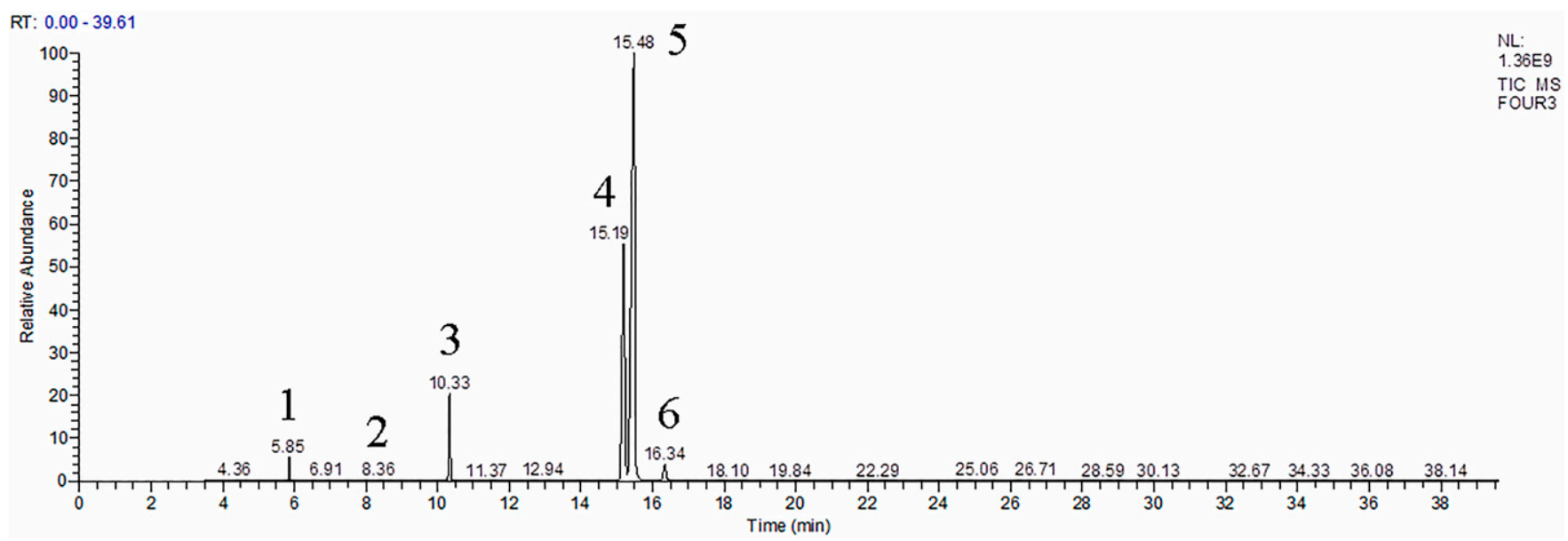

Fig. 5. Fatty acid methylester chromatogram for Paeonia ostii seed oil with the understory intercropping cultivation method. Numbers 1 to 6 indicate tridecanoic acid, oleic acid, palmitic acid, linoleic acid, $\alpha$-linolenic acid, and stearic acid, respectively.

\section{Discussion}

Effects of cultivation methods on the seed yield traits and oil yield of $P$. ostii. The growth and development of plants are easily influenced by the environmental conditions where they grow. Changes in environmental factors can cause different physiological and biochemical changes in plants. Many factors affected the oil-use features of $P$. ostii. For example, variety types, cultivation methods, geographical distribution, agronomic management measures, and habitat conditions are important factors affecting the oil-use features (Li, 2017). The effects of cultivation methods on the oiluse features of $P$. ostii were investigated from the three aspects of seed yield trait, oil yield, and fatty acid composition in this study. 


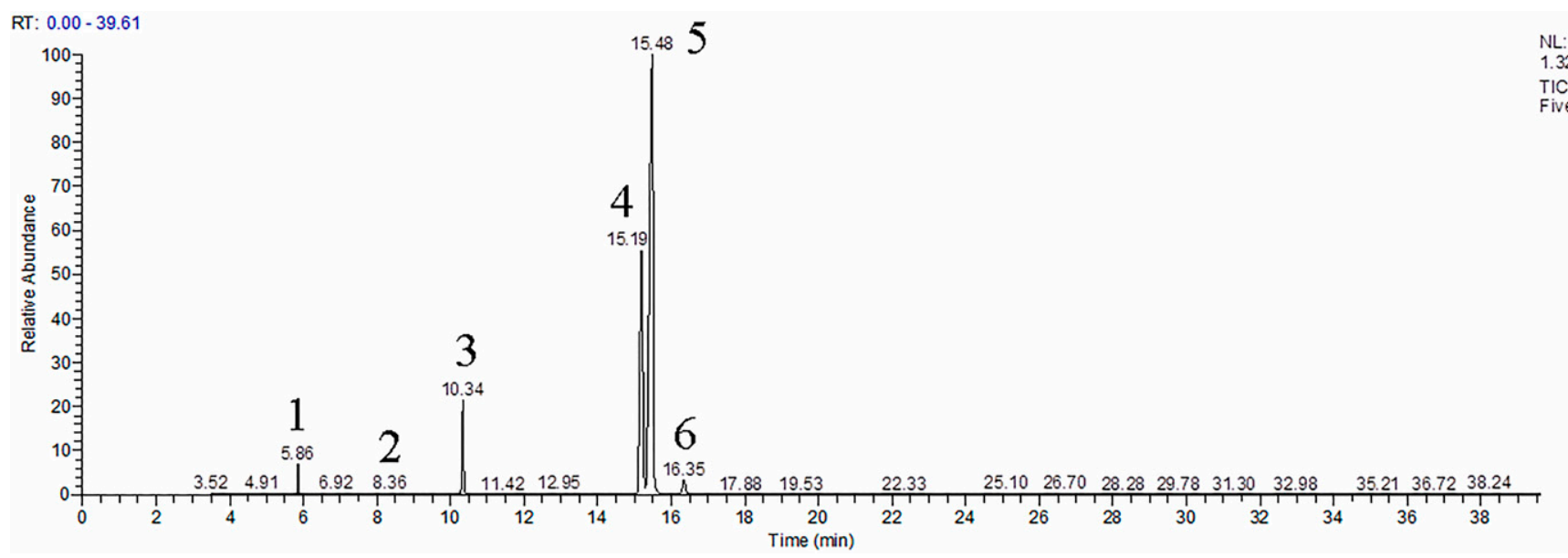

Fig. 6. Fatty acid methylester chromatogram for Paeonia ostii seed oil with the open field cultivation method. Numbers 1 to 6 indicate tridecanoic acid, oleic acid, palmitic acid, linoleic acid, $\alpha$-linolenic acid, and stearic acid, respectively.

Table 3. Differentiation of the fatty acid contents in seed oil of Paeonia ostii with different cultivation methods.

\begin{tabular}{lccccccc}
\hline $\begin{array}{l}\text { Cultivation } \\
\text { methods }\end{array}$ & $\begin{array}{c}\alpha \text {-Linolenic } \\
\text { acid (\%) }\end{array}$ & Linoleic acid (\%) & Oleic acid (\%) & Palmitic acid (\%) & Stearic acid (\%) & $\begin{array}{c}\text { Unsaturated } \\
\text { fatty acids (\%) }\end{array}$ & $\begin{array}{c}\text { Saturated fatty } \\
\text { acids (\%) }\end{array}$ \\
\hline $\begin{array}{c}\text { Sunny slope } \\
\text { cultivation }\end{array}$ & $40.94 \pm 0.03922 \mathrm{~b}$ & $17.35 \pm 0.01662 \mathrm{~d}$ & $0.00294 \pm 0.00028 \mathrm{a}$ & $3.64 \pm 0.00349 \mathrm{c}$ & $1.19 \pm 0.00114 \mathrm{c}$ & $58.3 \pm 0.05585 \mathrm{~d}$ & $4.83 \pm 0.00463 \mathrm{c}$ \\
$\begin{array}{c}\text { Shady slope } \\
\text { cultivation }\end{array}$ & $45.66 \pm 0.03451 \mathrm{a}$ & $18.28 \pm 0.01381 \mathrm{~b}$ & $0.0031 \pm 0.00023 \mathrm{a}$ & $3.98 \pm 0.003 \mathrm{a}$ & $1.29 \pm 0.00097 \mathrm{a}$ & $63.94 \pm 0.04832 \mathrm{c}$ & $5.26 \pm 0.00398 \mathrm{a}$ \\
$\begin{array}{c}\text { High-altitude } \\
\text { cultivation }\end{array}$ & $46.85 \pm 0.05765 \mathrm{a}$ & $17.72 \pm 0.02181 \mathrm{c}$ & $0.00263 \pm 0.00032 \mathrm{a}$ & $3.99 \pm 0.00491 \mathrm{a}$ & $1.12 \pm 0.00138 \mathrm{~d}$ & $64.57 \pm 0.07946 \mathrm{~b}$ & $5.11 \pm 0.00629 \mathrm{~b}$ \\
$\begin{array}{c}\text { Understory } \\
\text { intercropping } \\
\text { cultivation }\end{array}$ & $45.15 \pm 0.1858 \mathrm{a}$ & $20.08 \pm 0.08262 \mathrm{a}$ & $0.00233 \pm 0.00096 \mathrm{a}$ & $3.86 \pm 0.01589 \mathrm{~b}$ & $1.24 \pm 0.0051 \mathrm{~b}$ & $65.23 \pm 0.26843 \mathrm{a}$ & $5.1 \pm 0.02099 \mathrm{~b}$ \\
$\begin{array}{c}\text { Open field } \\
\text { cultivation }\end{array}$ & $37.19 \pm 0.20034 \mathrm{c}$ & $16.21 \pm 0.0873 \mathrm{e}$ & $0.0027 \pm 0.00015 \mathrm{a}$ & $3.28 \pm 0.01769 \mathrm{~d}$ & $0.89 \pm 0.00477 \mathrm{e}$ & $53.4 \pm 0.28766 \mathrm{~d}$ & $4.17 \pm 0.02246 \mathrm{~d}$ \\
\hline
\end{tabular}

Different lowercase letters in the same column represent significant differences among different treatments $(P<0.05)$.

The understory intercropping cultivation method yielded the best data for the six seed yield traits (fruit pod length, fruit pod width, fruit pod thickness, number of fruit pods per plant, 1000-grain weight, and seed yield per plant), followed by the high-altitude cultivation method, and no significant difference was found between the high-altitude cultivation method and understory intercropping cultivation method $(P<0.05)$. There were some obvious differences in the sizes of the individual plants with different cultivation conditions, which were probably attributable to the branching of the plant (Qin, 2016; Song et al., 2018; Zhang et al., 2020). The seed yield was closely related to the number of branches per plant (Zhang et al., 2020). Similarly, in this study, different cultivation conditions may have some effects on phenotypic traits of $P$. ostia, such as the branch. Our field investigation found that the individual size and number of branches of $P$. ostii were different with different cultivation methods, as was the number of flowers, which resulted in differences in the numbers and shapes of fruit pods per plant. Differences in the numbers and shapes of fruit pods could influence the seed yield of a single plant. These might explain why seed yield traits with the understory intercropping cultivation method were superior to the others. In further studies, the interactions between phenotypic traits and seed yield should be evaluated to determine more indicators, such as the number of flowers, the number of branches, plant height, and crown breadth, using the innovative methods and technologies in the fields of molecular biology, plant physiology and biochemistry, and multiomics.

The maximum oil yield $(30.41 \%)$ of $P$. ostii was reached with the understory intercropping cultivation method. It reached the secondary maximum value of $29.51 \%$ with the high-altitude cultivation method. However, the coefficient of oil yield variation (5.71) was minimal with the understory intercropping cultivation method, and it was more stable than that of the high-altitude cultivation. Therefore, understory intercropping cultivation is beneficial to increasing the seed yield and oil yield of $P$. ostii.

The understory intercropping system can reduce planting costs and management difficulty and improve the efficiency of land use and the economic benefits of land. Furthermore, it can effectively reduce the use of water, fertilizer, and humus. Previous studies have confirmed that understory intercropping cultivation can improve the seed yield of $P$. ostii. For example, Wang et al. (2013) found that $P$. ostii could increase the ventilation, light transmission, disease resistance, and stresstolerance with crabapple (Malus spectabilis) understory intercropping method, thus improving the seed yield and quality of $P$. ostii. P. ostii is a photophilous species that can grow better in places with sufficient light, but the high temperatures in summer weaken its physiological and biochemical characteristics, such as photosynthesis, respiration, and nutrient absorption, causing a reduction in seed production (Han et al., 2018). Similarly, in the present study, regarding planting $P$. ostii under the G. biloba tree, the G. biloba leaves in autumn can provide sufficient humus, and the tree canopy in summer can prevent much of the glare from solar radiation. This planting method can benefit the growth, development, and cycle of "sourceflow-library" for $P$. ostii, thus promoting the biomass increase and dry matter accumulation. Therefore, it can facilitate the understanding of why the cultivation pattern of "upper arbor and lower shrub" increased the seed yield and oil yield of $P$. ostii. In addition, after planting $P$. ostii under G. biloba, the soil produced a symbiotic fungi and other beneficial soil microbiome symbiosis with the roots of $P$. ostii. The activities of microorganisms and their metabolites could stimulate the roots of $P$. ostii to absorb soil nutrients and water as much as possible, which possibly resisted the external environment stress and enhanced photosynthesis and other physiological metabolic activities in P. ostii. 
Effects of cultivation methods on the fatty acid composition of seed oil of $P$. ostii. In the present study, the differential analysis of the fatty acid compositions with different cultivation methods showed that the total amount of unsaturated fatty acids (ALA, linoleic acid, and oleic acid) reached the maximum value of $65.23 \%$ with the understory intercropping cultivation method. Furthermore, the content of saturated fatty acids (palmitic acid and stearate acid) reached the maximum value of $5.26 \%$ with the shady slope cultivation method. The maximum content $(46.85 \%)$ of ALA was found with the high-altitude cultivation method. The maximum content of oleic acid $(0.0031 \%)$ was found with shady slope cultivation. The maximum content of linoleic acid was $20.08 \%$ with the understory intercropping cultivation method. In summary, the type and content of fatty acid compositions in $P$. ostii seed oil, especially the content of fatty acid compositions, displayed obviously different responses to different cultivation methods. It is perceived that highaltitude cultivation is conducive to high contents of functional-composition ALA. Therefore, altitude should be considered when estimating the oil-use features and quality of $P$. ostii and other oilseed crops.

Previous studies have demonstrated that altitude can promote the accumulation of the secondary metabolites in plants (Liu et al., 2018). For example, Li et al. (2018) collected Podophyllum hexdrum samples from two elevations $(2300 \mathrm{~m}$ and $3300 \mathrm{~m})$ as test materials, and the results showed that the analyte from the high elevation had high podophyllotxin contents, and the difference between the two elevations was close to three-times. In Leontodon species collected from different elevations, the flavonoids content had a significant positive correlation with elevation according to Zidorn and Stuppner (2001). Dong et al. (2011) observed a significant and positive correlation between the flavonoids content and elevation through an investigation of Eucommia ulmoides sampled from six different elevations of $550 \mathrm{~m}, 690 \mathrm{~m}, 780 \mathrm{~m}$, $845 \mathrm{~m}, 950 \mathrm{~m}$, and $1180 \mathrm{~m}(P<0.05)$. Furthermore, in the present study, the high-altitude cultivation method $(810 \mathrm{~m})$ involved a higher altitude than other cultivation methods, and the content of ALA was relatively high with this cultivation method. In highaltitude regions, full lighting and day-night temperature differences can enhance the photosynthesis of $P$. ostii, reduce respiratory consumption, and promote the synthesis and accumulation of unsaturated fatty acids, which perhaps effectively increased the contents of unsaturated fatty acids, especially ALA, consistent with the results reported by Wang et al. (2017) indicating that altitude increases can promote the accumulation of unsaturated fatty acids in seed oil of $P$. ostii.

Zhang et al. (2017a, 2018a) found rich flavonoids in $P$. ostii seeds. A special orthodihydroxylated structure exists in flavonoids substances that can absorb ultraviolet radiation and scavenge free radicals. High-altitude growth locations have strong ultraviolet radiation. The flavonoids contents increase with altitude, which is a long-term adaptation to the high-altitude environment, thus reflecting the reproductive strategy of the plant (Wulff et al., 1999). However, in other special plants, such as Buxus sempervirens L., the flavonoids contents decreased with increasing elevation (Bernal et al., 2013). The reduction of flavonoids in $B$. sempervirens leaves with altitude might result from the increased synthesis of wax in response to the decrease in temperature (Shepherd and Griffiths, 2006) because both cuticular waxes and flavonoids are derived from acetyl-CoA (Kunst and Samuels, 2003). Therefore, high altitude was conducive to the physiological and reproductive growth and the production of secondary metabolites of $P$. ostii, which may explain why unsaturated fatty acid composition contents are high in $P$. ostii seed oil with the high-altitude cultivation method. The mechanism of high elevation promoting the accumulation of unsaturated fatty acids remains unclear for this plant; this should be evaluated during a future study from the new perspectives of molecular biology and plant physiology combined with metabolomics, proteomics, and genomics. Only five cultivation methods were selected for this study; these cannot fully include all tree peony cultivation methods worldwide. More samples and test indicators need to be selected and investigated to thoroughly explore the most suitable cultivation method for $P$. ostii in the future to facilitate the improvement of its oil-use features for large-scale cultivation and application.

\section{Conclusions}

The six key traits of seed yield (fruit pod length, fruit pod width, fruit pod thickness, number of fruit pods per plant, 1000-grain weight, and seed yield per plant) of $P$. ostii were optimal with the understory intercropping cultivation method. The highest oil yield and the highest total amount of unsaturated fatty acids were obtained with this cultivation method as well. The high-altitude cultivation method promoted the accumulation of the functional ingredient, ALA. Based on these results, a feasible and suitable cultivation approach was proposed for high-yield and high-quality seed oil harvesting. Therefore, considering the oiluse features of $P$. ostii, the understory intercropping cultivation and appropriate altitude increases are recommended as a preferable cultivation method for this plant.

\section{Literature Cited}

Bai, Z.Z., Y.L. Zhang, R. Yu, J. Li, and L.X. Niu. 2017. Comparison of different extraction methods for seed oil from the 'Fengdan' peony cultivar. Food Sci. 38:136-141, https://doi.org/ 10.7506/spkx1002-6630-201701022.

Bernal, M., L. Llorens, R. Julkunen-Tiitto, J. Badosac, and D. Verdaguer. 2013. Altitudinal and seasonal changes of phenolic compounds in Buxus sempervirens leaves and cuticles. Plant Physiol. Biochem. 70:471-482, https:// doi.org/10.1016/j.plaphy.2013.06.012. de Andrade, L.C., R. Andreazza, and F.A. de Oliveira Camargo. 2019. Cultivation of sorghum and sunflower in soils with amendment of sludge from industrial landfill. Intl. J. Recycl. Org. Waste Agr. 8:119-130, https://doi.org/ 10.1007/s40093-018-0236-4.

Dong, J.E., X.H. Ma, Q. Wei, S.B. Peng, and S.C. Zhang. 2011. Effects of growing location on the contents of secondary metabolites in the leaves of four selected superior clones of Eucommia ulmoides. Ind. Crops Prod. 34:1607-1614, https://doi.org/10.1016/j.indcrop.2011.06.007.

Duan, X.G., L.X. Zhang, W. Liu, L.L. Guo, D.L. Guo, and X.G. Hou. 2018. Effects of nitrogen application on photosynthetic characteristics and yields of oil tree peony Paeonia ostii 'Fengdan'. J. Nanjing For. Univ. (Natural Science Edition) 42:48-54, https://doi.org/10.3969/j.issn.10002006.201704033.

Flora of China Editorial Committee. 2001. Flora of China. 1st ed. Science Press, Beijing, and Missouri Botanical Garden Press, St. Louis.

Gao, T.T., Y.Y. Wang, and J.W. Ren. 2013. Analysis of the composition in peony seed oil by GC/MS and evaluation in the sunscreen effect. Food Sci. Technol. (Campinas) 38:1-4, https:// doi.org/10.13684/j.cnki.spkj.2013.06.021.

Han, C.J., Q. Wang, H.B. Zhang, S.H. Wang, H.D. Song, J.M. Hao, and H.Z. Dong. 2018. Light shading improves the yield and quality of seed in oil-seed peony (Paeonia ostii Feng Dan). J. Integr. Agr. 17:1631-1640, https://doi.org/ 10.1016/S2095-3119(18)61979-3.

Han, J.G., X.Q. Li, Z. Liu, and Y.H. Hu. 2014 Potential applications of tree peony as an oil plant. Cereals and Oils 27:21-25, https://doi. org/10.3969/j.issn.1008-9578.2014.05.006.

Han, J.G., Z. Liu, X.Q. Li, J. Li, and Y.H. Hu. 2016. Diversity in seed oil content and fatty acid composition in three tree peony species with potential as sources of omega-3 fatty acids. J. Hort. Sci. Biotechnol. 91:175-179, https://doi.org/10.1080/ 14620316.2015.1133538.

Konuskan, D.B. and B. Mungan. 2016. Effects of variety, maturation and growing region on chemical properties, fatty acid and sterol compositions of virgin olive oils. J. Amer. Oil Chem. Soc. 93: 1499-1508, https://doi.org/10.1007/s11746-0162904-8.

Konuskan, D.B., M. Arslan, and A. Oksuz. 2019. Physicochemical properties of cold pressed sunflower, peanut, rapeseed, mustard and olive oils grown in the Eastern Mediterranean region. Saudi J. Biol. Sci. 26:340-344, https://doi.org/ 10.1016/j.sjbs.2018.04.005.

Kunst, L. and A.L. Samuels. 2003. Biosynthesis and secretion of plant cuticular wax. Prog. Lipid Res. 42:51-80, https://doi.org/10.1016/ S0163-7827(02)00045-0.

$\mathrm{Li}, \mathrm{C.C}$. 2017. Influence to yield and quality of Paeonia ostii 'Fengdan' caused by different kinds of micronutrient fertilizer. Northwest Agriculture \& Forestry University, Shanxi, China.

Li, J.J. 2011. Tree Peony of China. 1st ed. Encyclopedia of China Publishing House, Beijing, China.

Li, M.F., L. Ge, T.L. Kang, P. Sun, H. Xing, D.L. Yang, J.L. Zhang, and P.W. Paréb. 2018. High-elevation cultivation increases anti-cancer podophyllotoxin accumulation in Podophyllum hexandrum. Ind. Crops Prod. 121:338-344, https://doi.org/10.1016/j.indcrop.2018.05.036.

Lin, C., H.Y. Lin, J.H. Chen, W.P. Tseng, P.Y. Ko, Y.S. Liu, W.L. Yeh, and D.Y. Lu. 2015. Effects of paeonol on anti-neuroinflammatory responses in microglial cells. Intl. J. Mol. Sci. 16:8844 8860, https://doi.org/10.3390/ijms16048844. 
Liu, W., D.M. Wang, X.G. Hou, Y.Q. Yang, X. Xue, Q.S. Jia, L.X. Zhang, W. Zhao, and D.X. Yin. 2018. Effects of growing location on the contents of main active components and antioxidant activity of Dasiphora fruticosa (L.) Rydb. by chemometric methods. Chem. Biodivers. 15:E1800114, https://doi.org/10.1002/cbdv.201800114.

Luo, J.R., Y.L. Zhang, L.P. Guo, and L.X. Niu. 2016. Evaluation study on the seed oil features of 35 cultivated tree peony varieties. J. Chinese Cereals and Oils Assn. 31:60-65, https://doi. org/10.3969/j.issn.1003-0174.2016.10.011.

Mariela, T., P. Pierluigi, S. Peter, R. Cecilia, G. García-Inza, A. Miserere, R. Bodoira, C. Contreras, and D. Maestri. 2017. Olive cultivation in the southern hemisphere: Flowering, water requirements and oil quality responses to new crop environments. Front. Plant Sci. 8:1830-1839, https:// doi.org/10.3389/fpls.2017.01830.

Nichols, P.D., J. Petrie, and S. Singh. 2010. Longchain omega-3 oils: An update on sustainable sources. Nutrients 2:572-585, https://doi.org/ $10.3390 /$ nu2060572.

Qin, W. 2016. Influence of different factors on cultivated quality of tree peony as an oil plant-A case study of Paeonia ostii. Northeast Forestry University, Haerbin, China.

Shepherd, T. and W. Griffiths. 2006. The effects of stress on plant cuticular waxes. New Phytol. 171: 469-499, https://doi.org/10.1111/j.1469-8137. 2006.01826.x.

Shi, G.A., F.X. Jiao, Y.P. Jiao, H.A. Yang, M.W. Han, Y.Q. Wu, and B.R. Shi. 2014. Development prospects and strategies of oil tree peony industry in China. J. Chinese Cereals and Oils Assn. 29:124-128, https://doi.org/10.3969/j. issn.1003-0174.2014.09.025.

Song, H.W., S.H. Liu, Z.G. Shen, G.J. Yuan, and Z.Y. Wang. 2018. Seed yields and oil contents of oil peony under different cultivation conditions.
Nonwood Forest Research 36:105-109, https:// doi.org/10.14067/j.cnki.1003-8981.2018.01.017.

Wang, S.L., X.X. Ren, J.Q. Xue, and X.X. Zhang. 2016. Research progress in the competent, function and process technology of peony seed oil. J. Chinese Cereals and Oils Assn. 31:139-147, https:// doi.org/10.3969/j.issn.1003-0174.2016.03.027.

Wang, W.W., S.L. Wu, H. Liao, K.J. Zhou, F. Liu, and J. Zhou. 2017. Effects of altitude and cultivation density on yield and quality of oil tree peony. South China Agr. 11:73-76, https://doi. org/10.19415/j.cnki.1673-890x.2017.34.020.

Wang, Z.R., X. Gu, X.X. Li, and J.F. Wang. 2013. Oil peony intercropping technology. J. Shandong For. Sci. Technol. 43:87-89, https://doi. org/10.3969/j.issn.1002-2724.2013.04.024.

Wei, X.B., J.Q. Xue, S.L. Wang, Y.Q. Xue, H. Lin, X.F. Shao, D.H. Xu, and X.X. Zhang. 2018. Fatty acid analysis in the seeds of 50 Paeonia ostii individuals from the same population. J. Integr. Agr. 17:1758-1767, https://doi. org/10.1016/S2095-3119(18)61999-9.

Wulff, A., S. Anttonen, R. Pellinen, E.M. Savonen, M.L. Sutinen, W. Heller, H. Sandermann, Jr., and J. Kangasjärvi. 1999. Birch (Betula pendula Roth) responses to high UV-B radiation. Boreal Environ. Res. 4:77-88.

Xue, J.Q., S.L. Wang, P. Zhang, F.Y. Zhu, X.X. Ren, C.J. Liu, and X.X. Zhang. 2015. On the role of physiological substances, abscisic acid and its biosynthetic genes in seed maturation and dormancy of tree peony (Paeonia ostii 'Fengdan'). Scientia Hort. 182:92-101, https:// doi.org/10.1016/j.scienta.2014.11.021.

Zhang, L.X., M.Y. Li, D.F. Wei, Q.S. Chang, X.G. Hou, B.H. Zhou, B.S. Lou, and H. Li. 2018 b. Effects of balanced fertilization on growth and seed yield of Paeonia ostii cv. 'Fengdan'. J. Gansu Agr. Univ. 53:58-68, https://doi.org/ 10.13432/j.cnki.jgsau.2018.05.009.
Zhang, Q.Y., L.X. Niu, R. Yu, X.X. Zhang, Z.Z. Bai, K. Duan, Q.H. Gao, and Y.L. Zhang. 2016. Cloning, characterization, and expression analysis of a gene encoding a putative lysophosphatidic acid acyltransferase from seeds of Paeonia rockii. Appl. Biochem. Biotechnol. 182:721-741, https://doi.org/10.1007/s12010-016-2357-4.

Zhang, X.X., J.Y. Sun, L.X. Niu, and Y.L. Zhang. 2017b. Chemical compositions and antioxidant activities of essential oils extracted from the petals of three wild tree peony species and eleven cultivars. Chem. Biodivers. 14:E1700282, https://doi.org/10.1002/cbdv.201700282.

Zhang, X.X., Q.Q. Shi, D. Ji, L.X. Niu, and Y.L. Zhang. 2017a. Determination of the phenolic content, profile, and antioxidant activity of seeds from nine tree peony (Paeonia section Moutan DC.) species native to China. Food Res. Intl. 97:141-148, https://doi.org/10.1016/j. foodres.2017.03.018.

Zhang, X.X., Y.L. Zhang, L.X. Niu, J.Y. Sun, L.H. Li, J. Zhang, and J. Li. 2017c. Chemometric classification of different tree peony species native to China based on the assessment of major fatty acids of seed oil and phenotypic characteristics of the seeds. Chem. Biodivers. 14:E1600111, https://doi.org/10.1002/cbdv.201600111.

Zhang, X.X., Z. Gang, M. Jin, L.X. Niu, and Y.L. Zhang. 2018a. Variation in phenolic content, profile, and antioxidant activity of seeds among different Paeonia ostii cultivated populations in China. Chem. Biodivers. 15:E1800093, https:// doi.org/10.1002/cbdv.201800093.

Zhang, Y.L., L.X. Niu, Q.Y. Zhang, and X.X. Zhang. 2020. China Peony Germplasm Resource. China Forestry Publishing House, Beijing.

Zidorn, C. and H. Stuppner. 2001. Evaluation of chemosystematic characters in the genus Leontodon (Asteraceae). Taxon 50:115-133, https:// doi.org/10.2307/1224515. 
ONE2 \#694 RT: 5.86 AV: 1 AV: 5 SB: 12 687-692 696-701 NL: 1.44E7

$\mathrm{T}:+\mathrm{c}$ El Full ms [30.00-460.00]

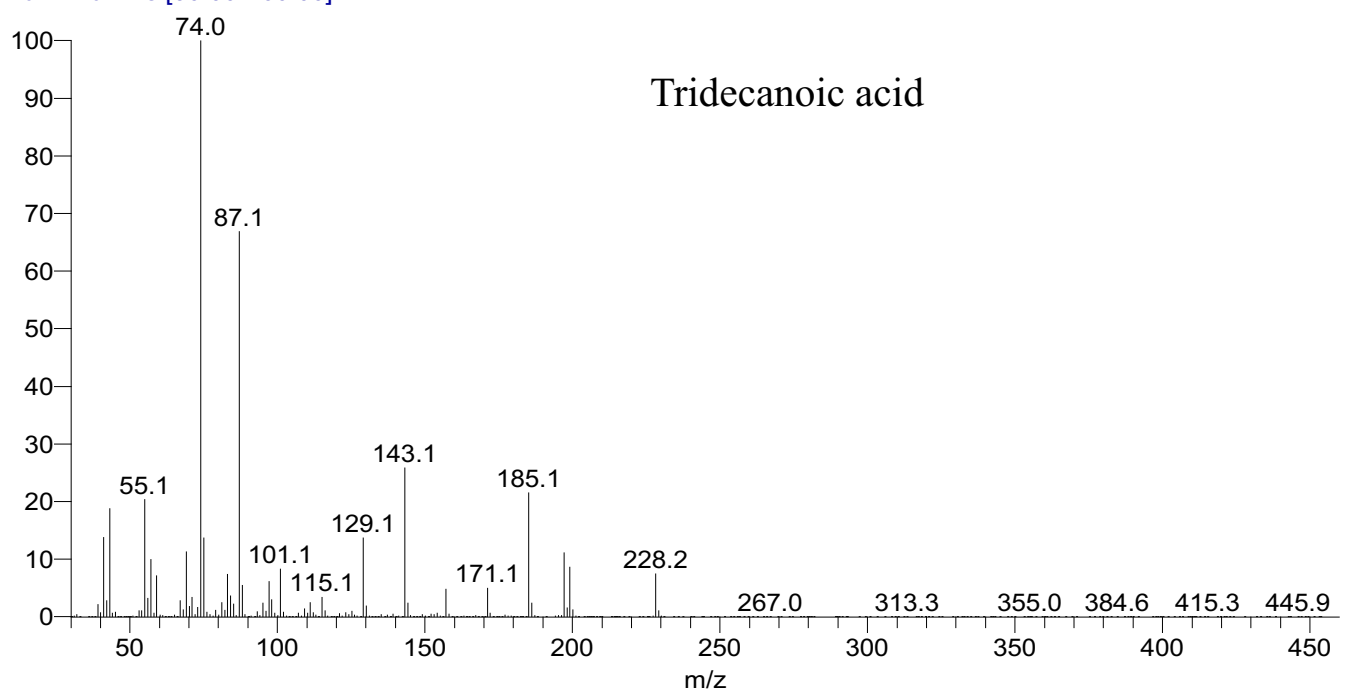

ONE2 \#1430 RT: 8.36 AV: 1 AV: 5 SB: 12 1423-1428 1432-1437 NL: 7.32E4

T: + c El Full ms [30.00-460.00]

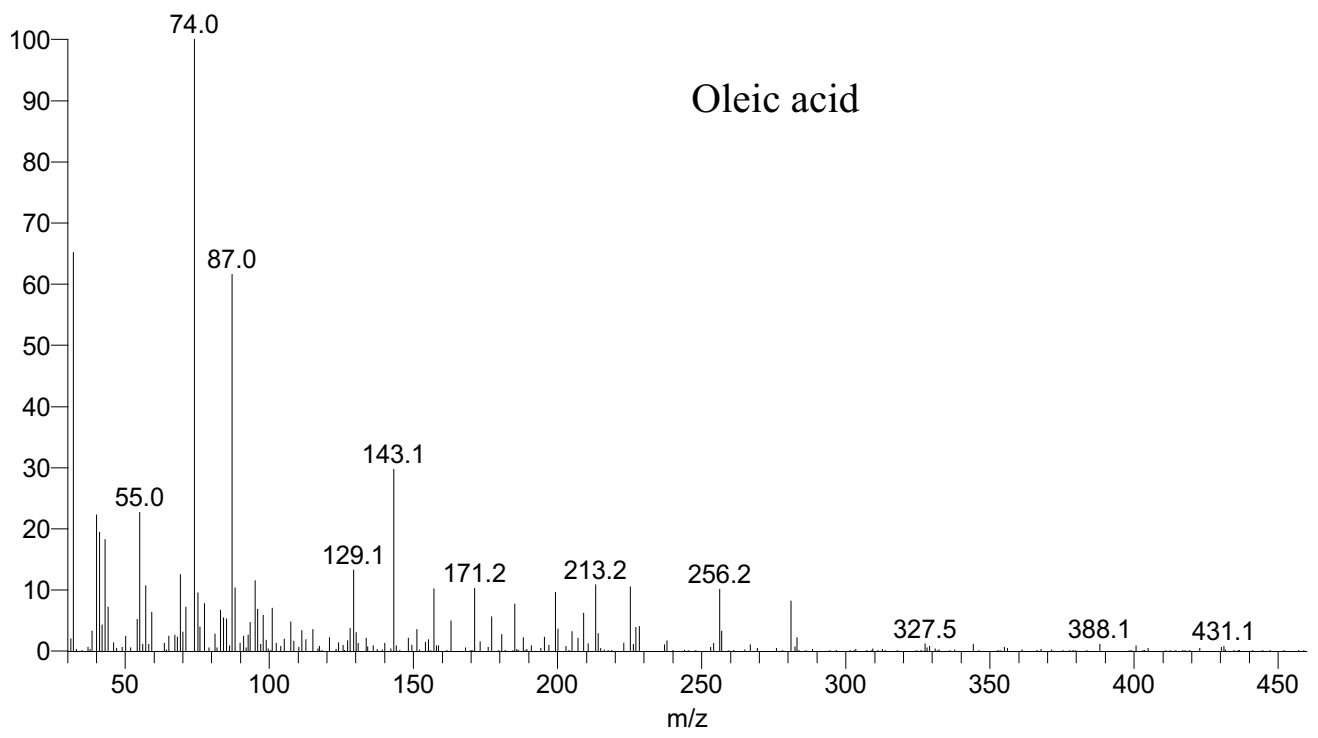

ONE2 \#2012 RT: 10.34 AV: 1 AV: 5 SB: 12 2005-2010 2014-2019 NL: 5.46E7

$\mathrm{T}:$ + c El Full ms [30.00-460.00]

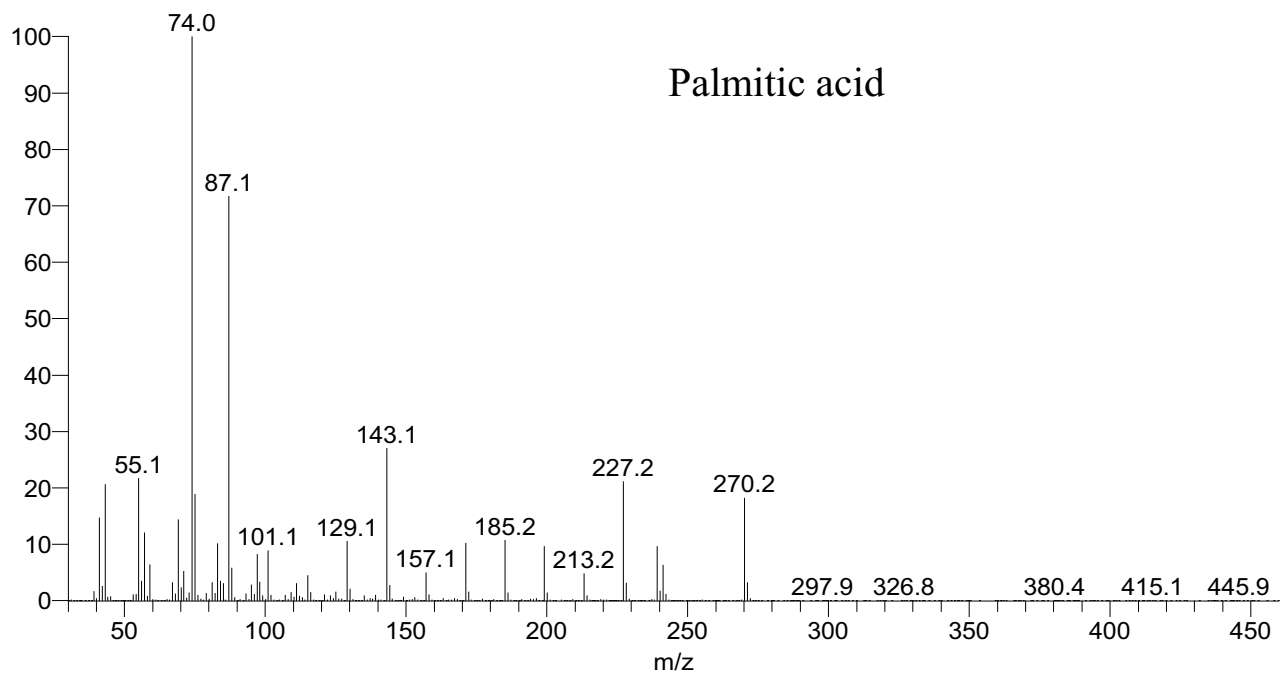

Supplemental Fig. 1. Ion map of six main fatty acids in Paeonia ostii seed oil with the sunny slope cultivation method. 
ONE2 \#3443 RT: 15.21 AV: 1 AV: 5 SB: 12 3436-3441 3445-3450 NL: 5.65E7

$\mathrm{T}:$ + c El Full ms [30.00-460.00]

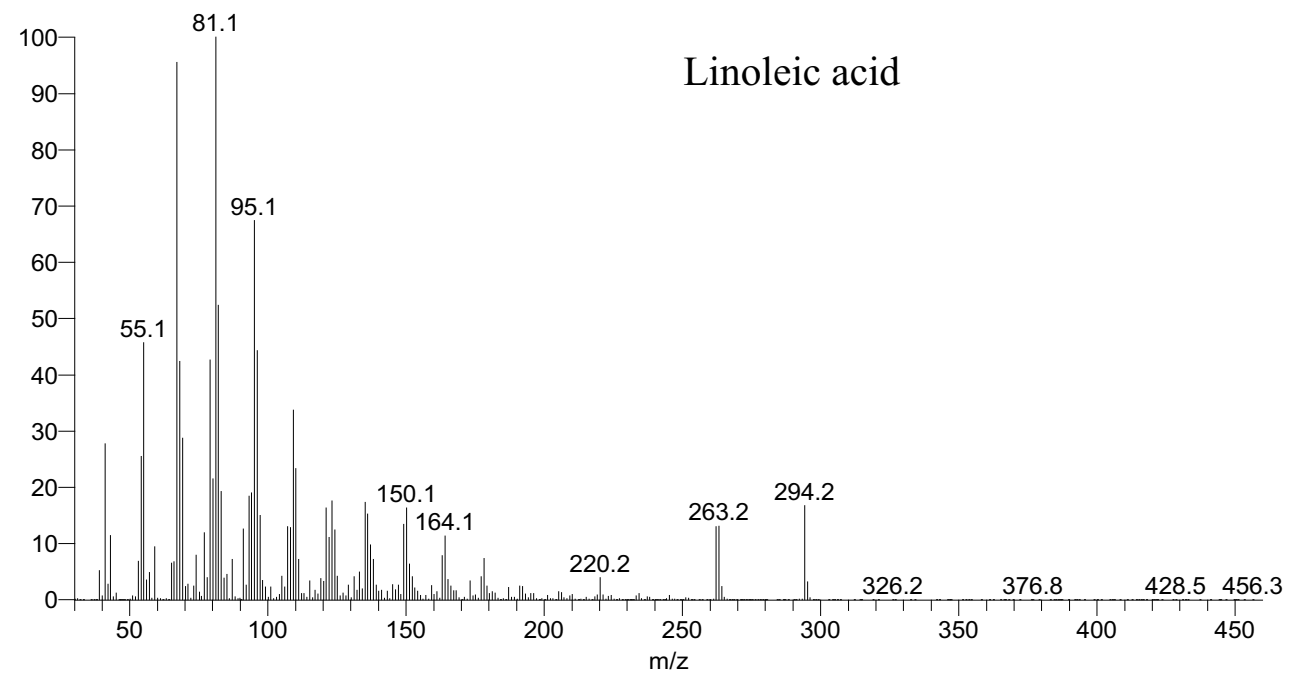

ONE2 \#3529 RT: 15.50 AV: 1 AV: 5 SB: 12 3522-3527 3531-3536 NL: 1.17E8

$\mathrm{T}:$ + c El Full ms [30.00-460.00]

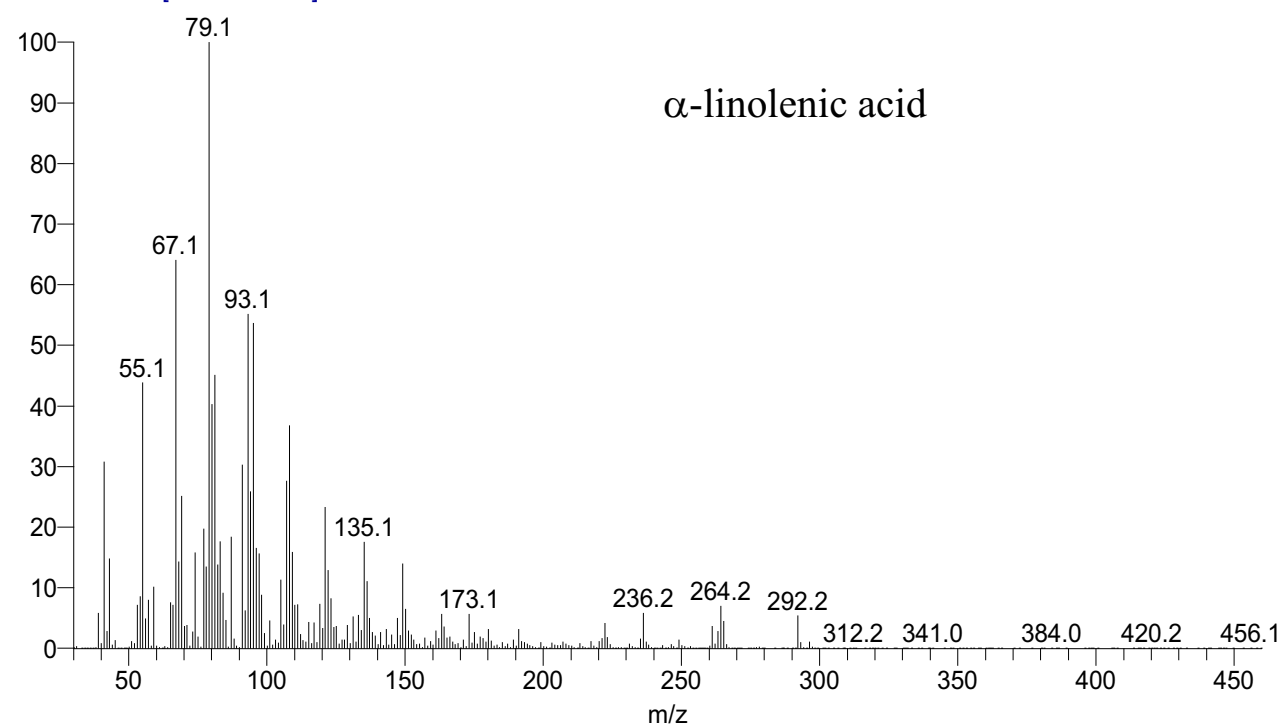

ONE2 \#3781 RT: 16.36 AV: 1 AV: 5 SB: 12 3774-3779 3783-3788 NL: 9.60E6 $\mathrm{T}:$ + c El Full ms [30.00-460.00]

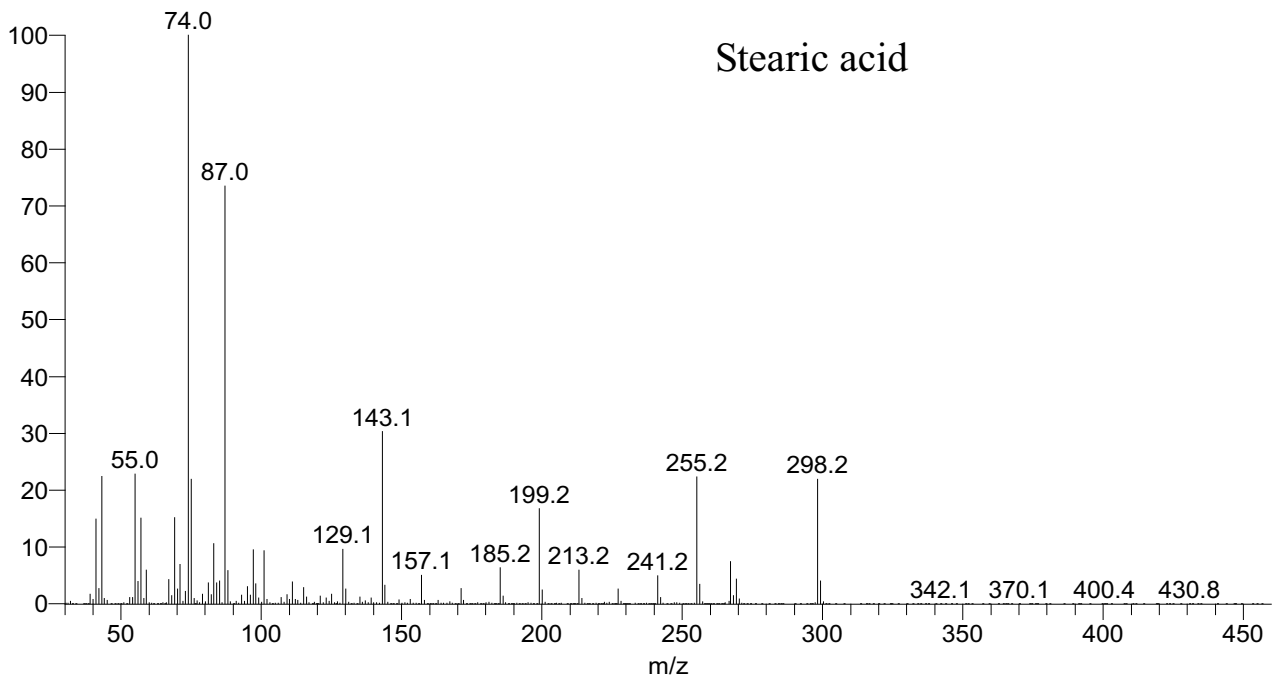

Supplemental Fig. 1. (Continued). 
ONE2 \#3443 RT: 15.21 AV: 1 AV: 5 SB: 12 3436-3441 3445-3450 NL: 5.65E7

$\mathrm{T}:$ + c El Full ms [30.00-460.00]

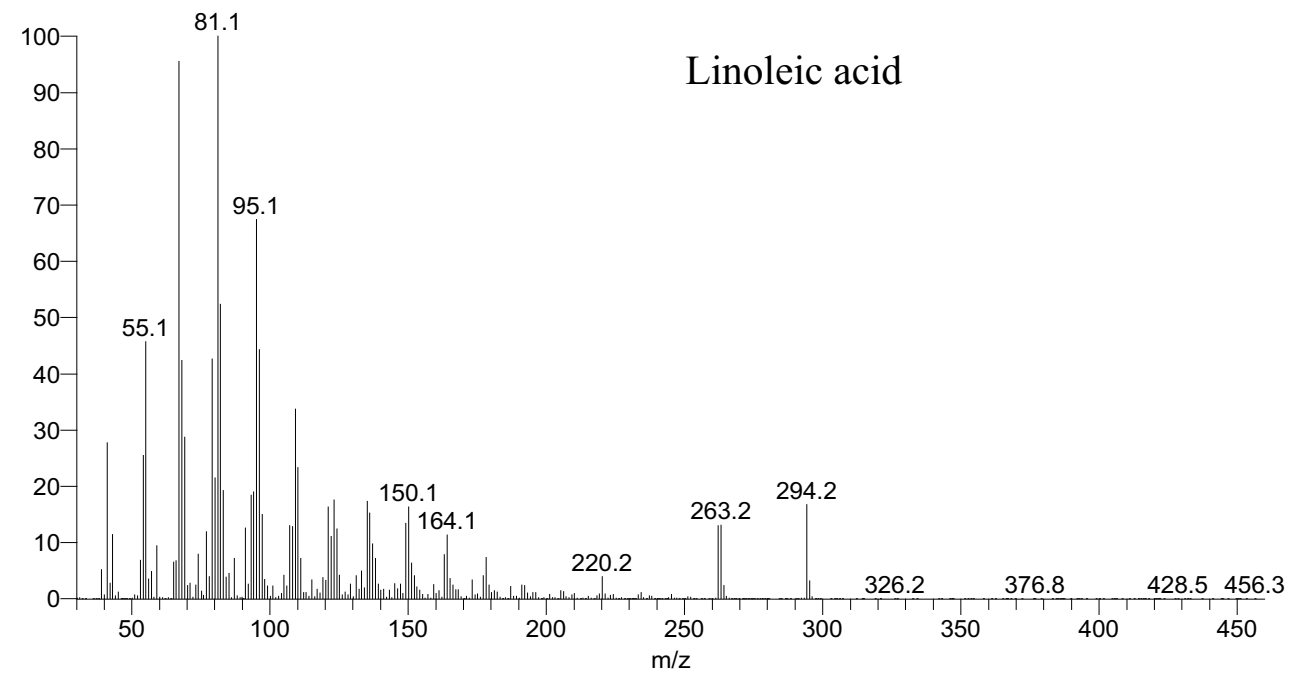

ONE2 \#3529 RT: 15.50 AV: 1 AV: 5 SB: 12 3522-3527 3531-3536 NL: 1.17E8

T: + c El Full ms [30.00-460.00]

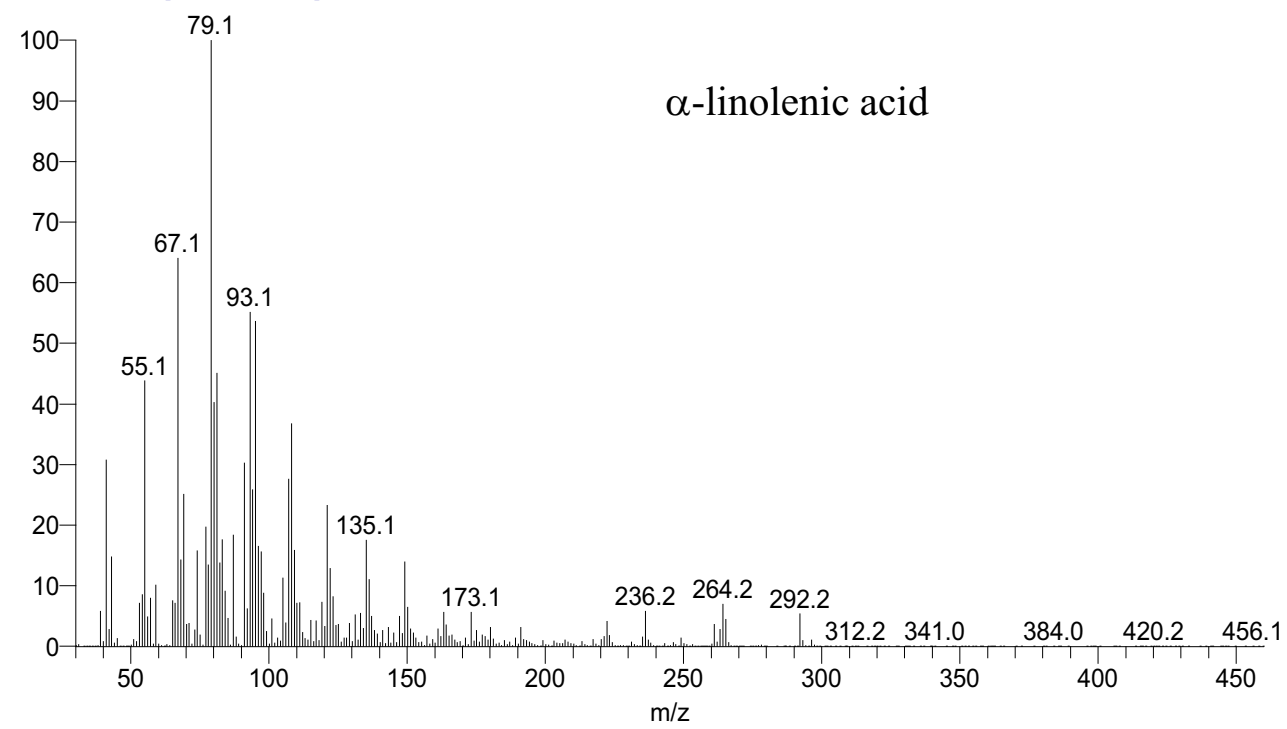

ONE2 \#3781 RT: $16.36 \quad$ AV: 1 AV: 5 SB: 12 3774-3779 3783-3788 NL: 9.60E6

$\mathrm{T}:+\mathrm{c}$ El Full ms [30.00-460.00]

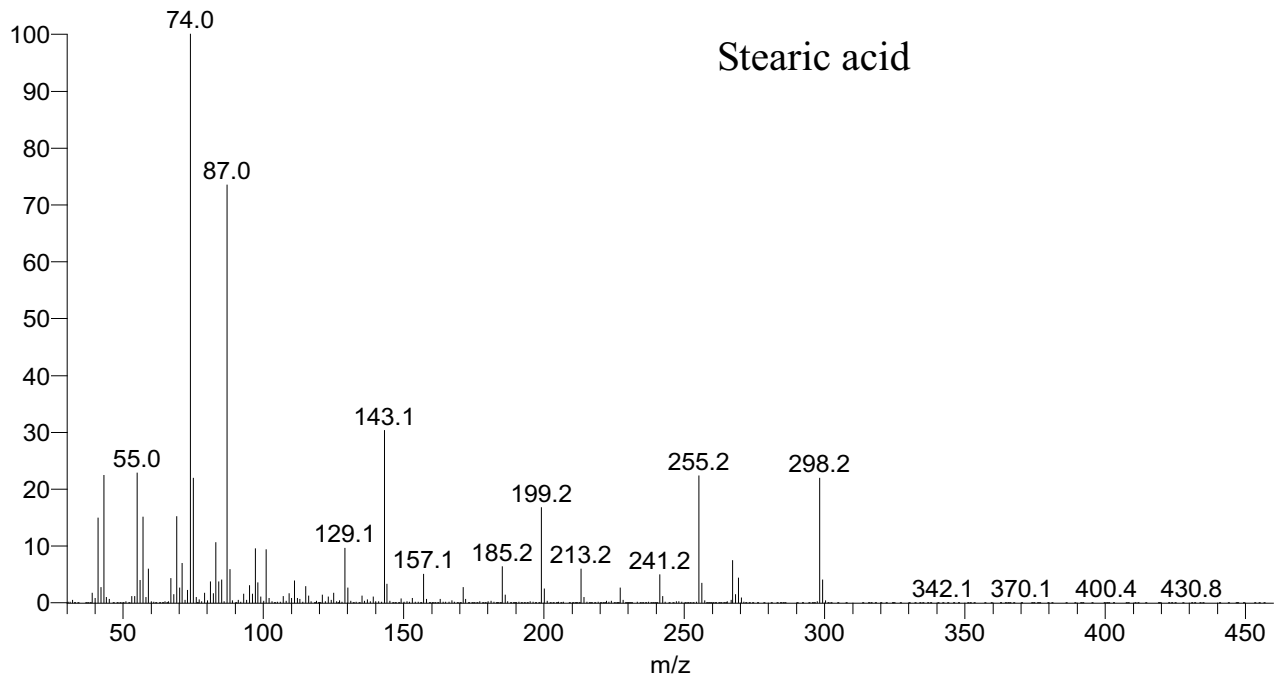

Supplemental Fig. 2. Ion map of six main fatty acids in Paeonia ostii seed oil with the shady slope cultivation method. 
TWO2 \#3441 RT: 15.20 AV: 1 AV: 5 SB: 12 3434-3439 3443-3448 NL: 5.93E7 T: + c El Full ms [30.00-460.00]

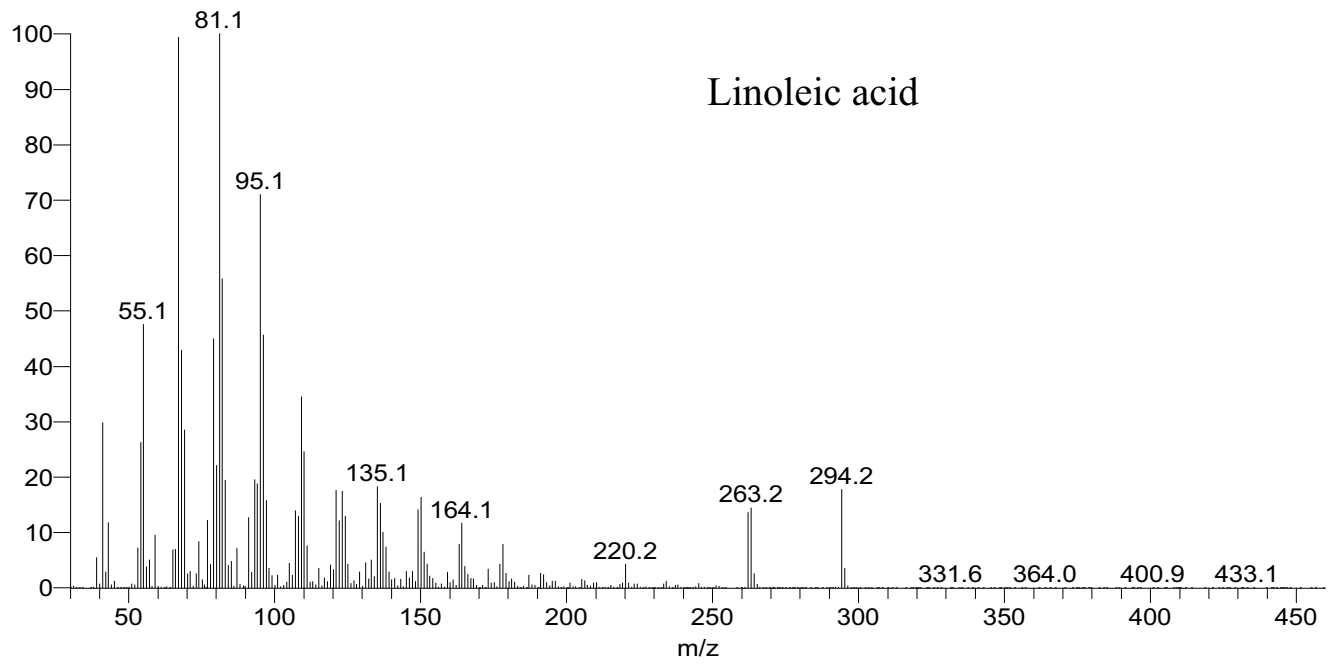

TWO2 \#3525 RT: 15.49 AV: 1 AV: 5 SB: 12 3518-3523 3527-3532 NL: $1.12 E 8$ $\mathrm{T}:+\mathrm{c}$ El Full ms [30.00-460.00]

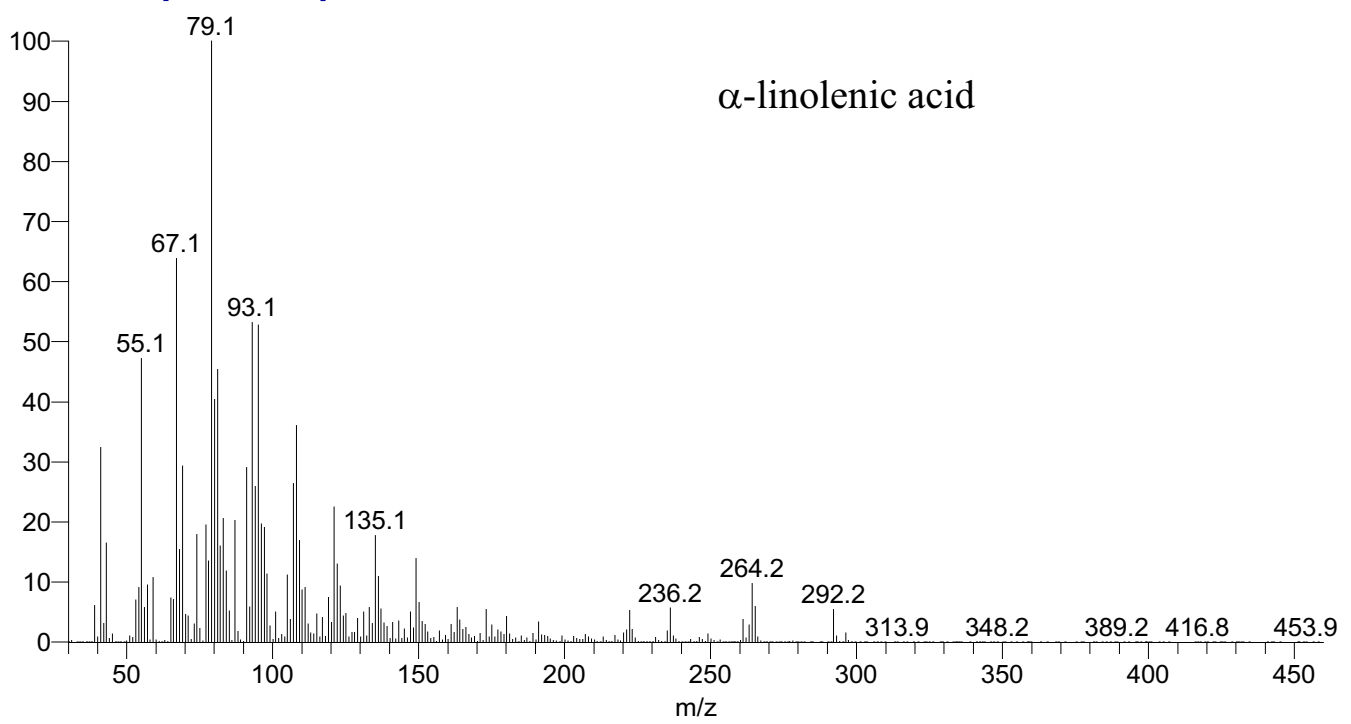

TWO2 \#3778 RT: 16.35 AV: 1 AV: 5 SB: 12 3771-3776 3780-3785 NL: $9.61 \mathrm{E} 6$

$\mathrm{T}:$ + c El Full ms [30.00-460.00]

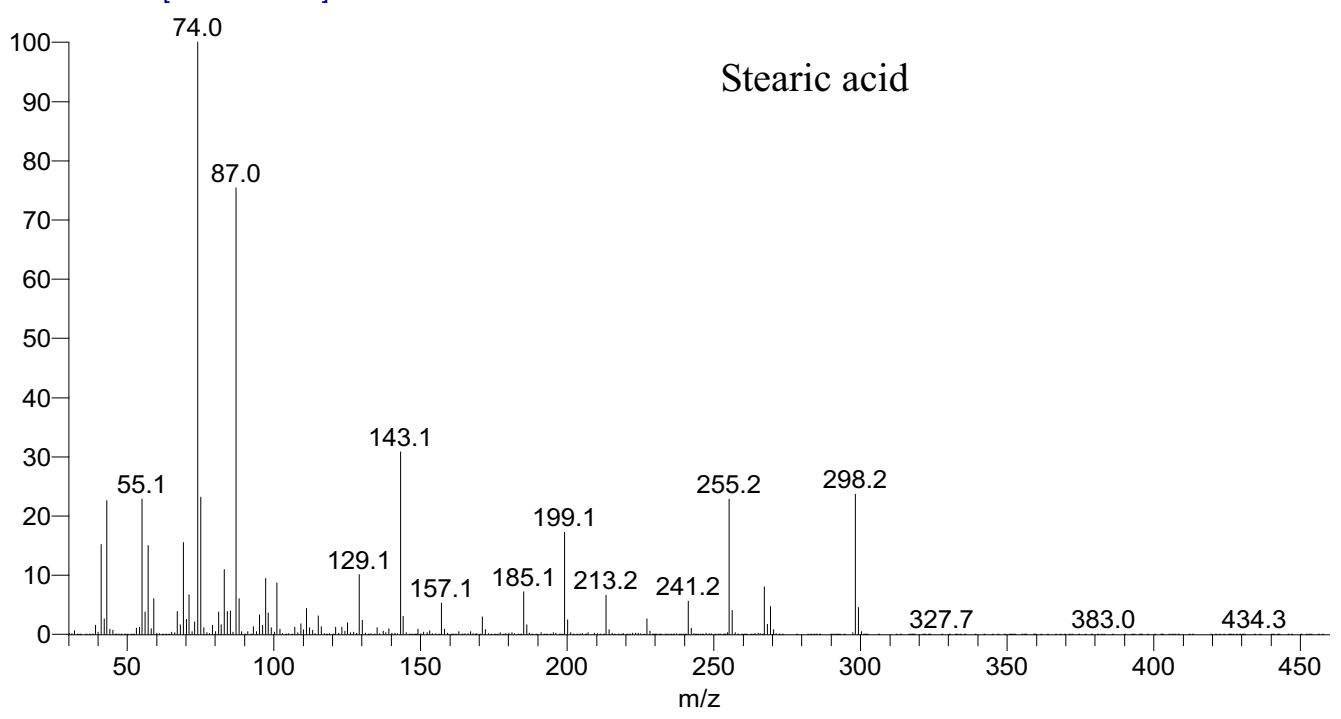

Supplemental Fig. 2. (Continued). 
THREE4 \#693 RT: 5.85 AV: 1 AV: 5 SB: 12 686-691 695-700 NL: 1.42E7

T: + c El Full ms [30.00-460.00]

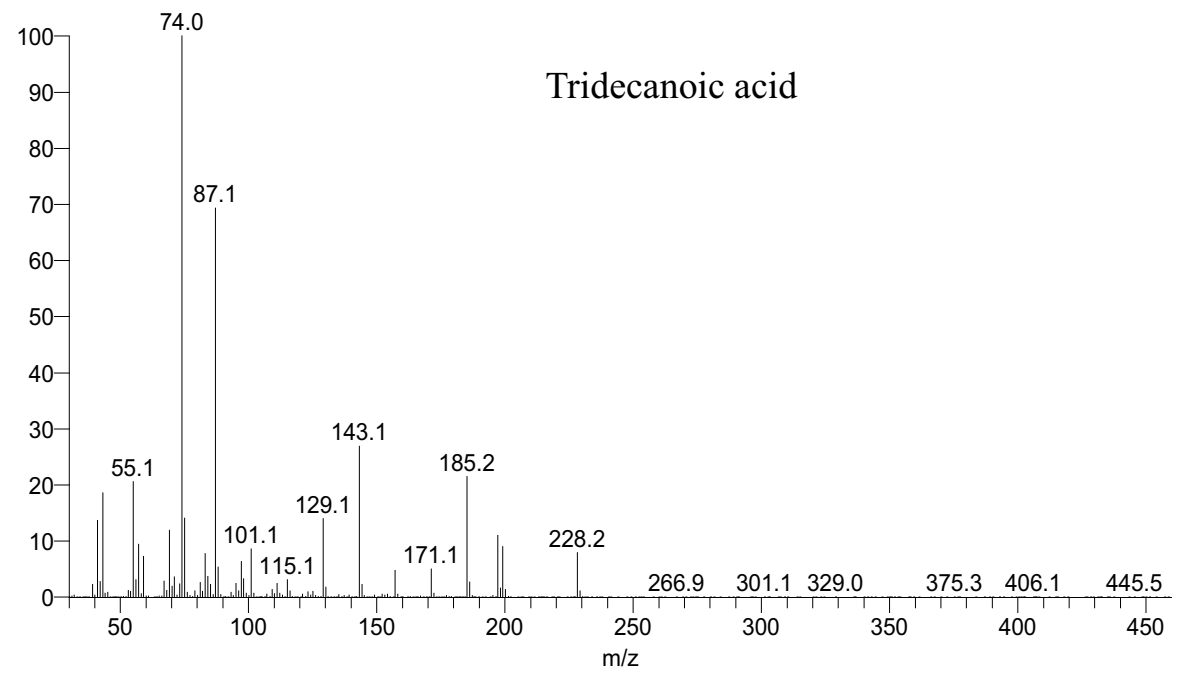

THREE4 \#1432 RT: 8.37 AV: 1 AV: 5 SB: 12 1425-1430 1434-1439 NL: 7.50E4

T: + c El Full ms [30.00-460.00]

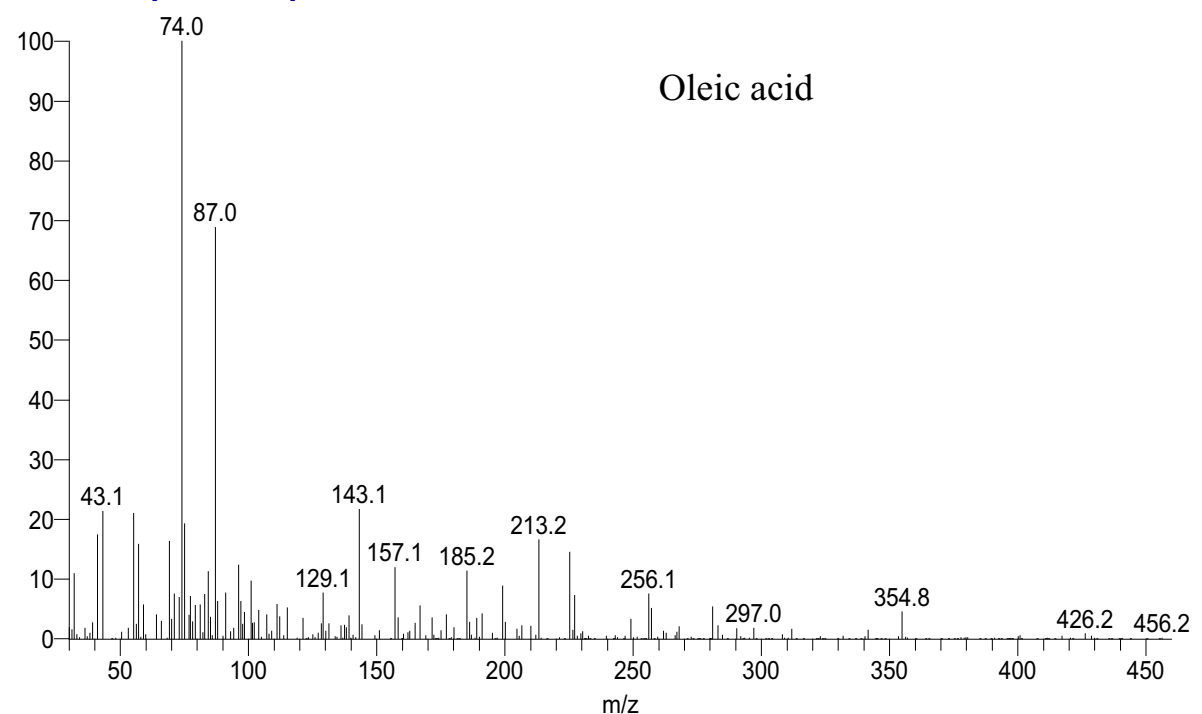

THREE4 \#2010 RT: 10.33 AV: 1 AV: 5 SB: 12 2003-2008 2012-2017 NL: 5.16E7 $\mathrm{T}:+\mathrm{c}$ El Full ms [30.00-460.00]

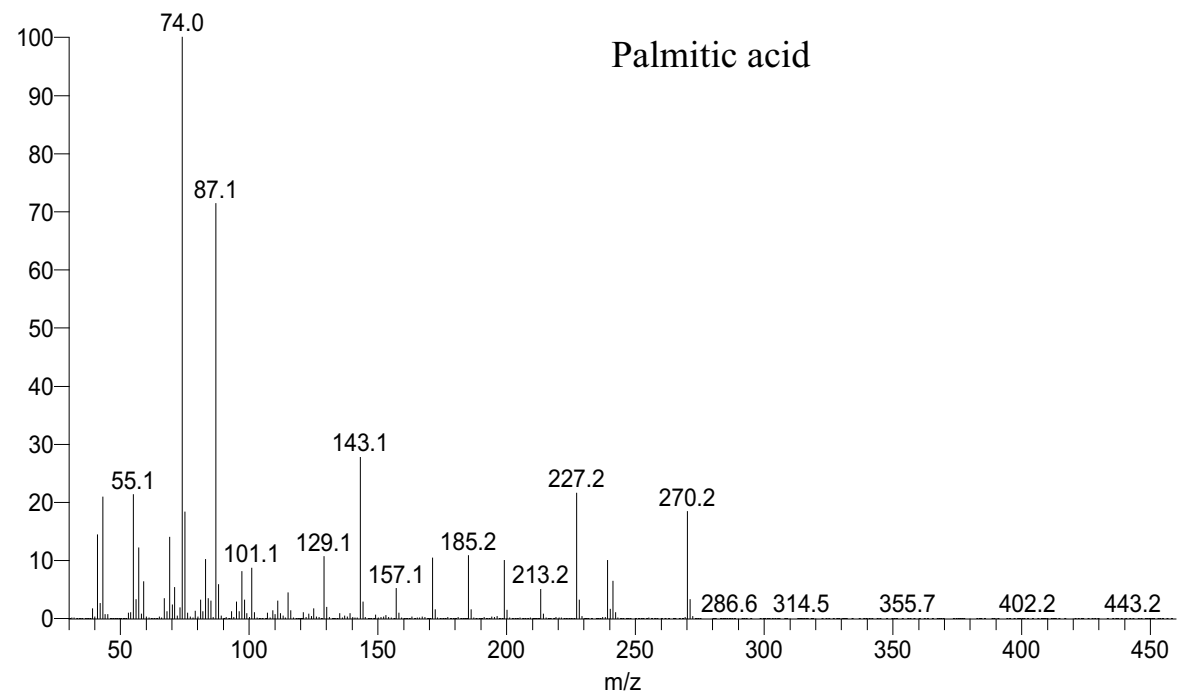

Supplemental Fig. 3. Ion map of six main fatty acids in Paeonia ostii seed oil with the high-altitude cultivation method. 
THREE4 \#3438 RT: 15.19 AV: 1 AV: 5 SB: 12 3431-3436 3440-3445 NL: 5.43E7

$\mathrm{T}:$ + c El Full ms [30.00-460.00]

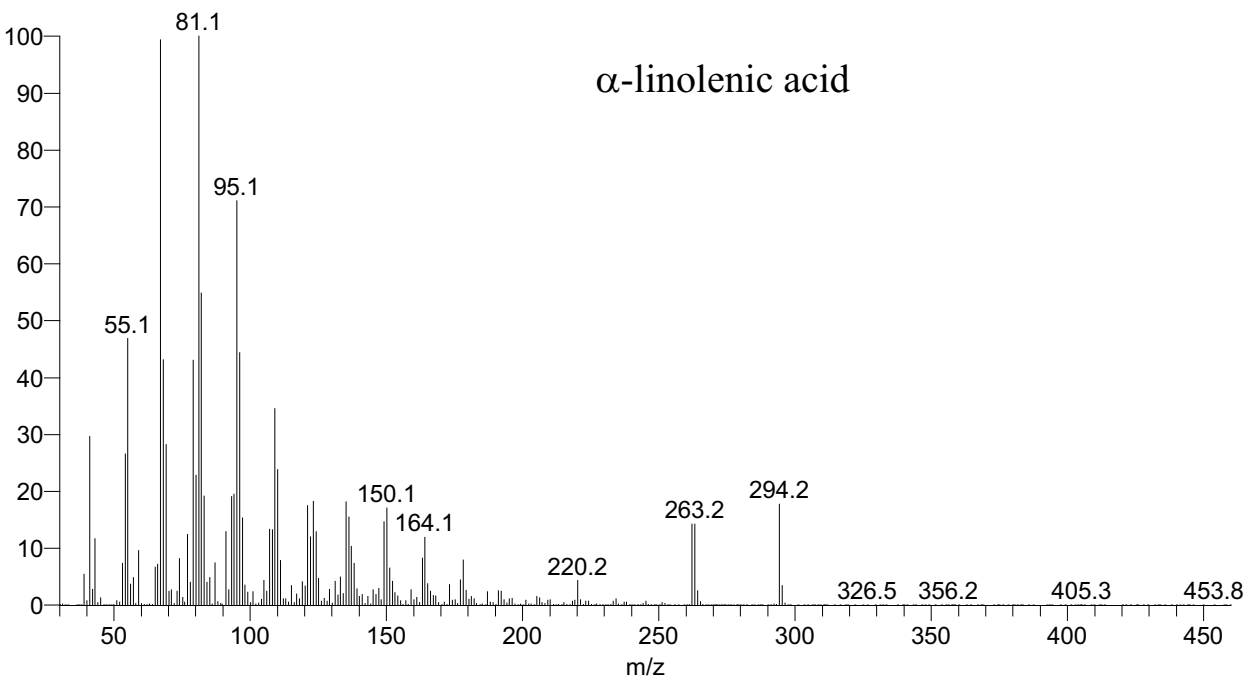

THREE4 \#3526 RT: 15.49 AV: 1 AV: 5 SB: 12 3519-3524 3528-3533 NL: 1.28E8 T: + c El Full ms [30.00-460.00]

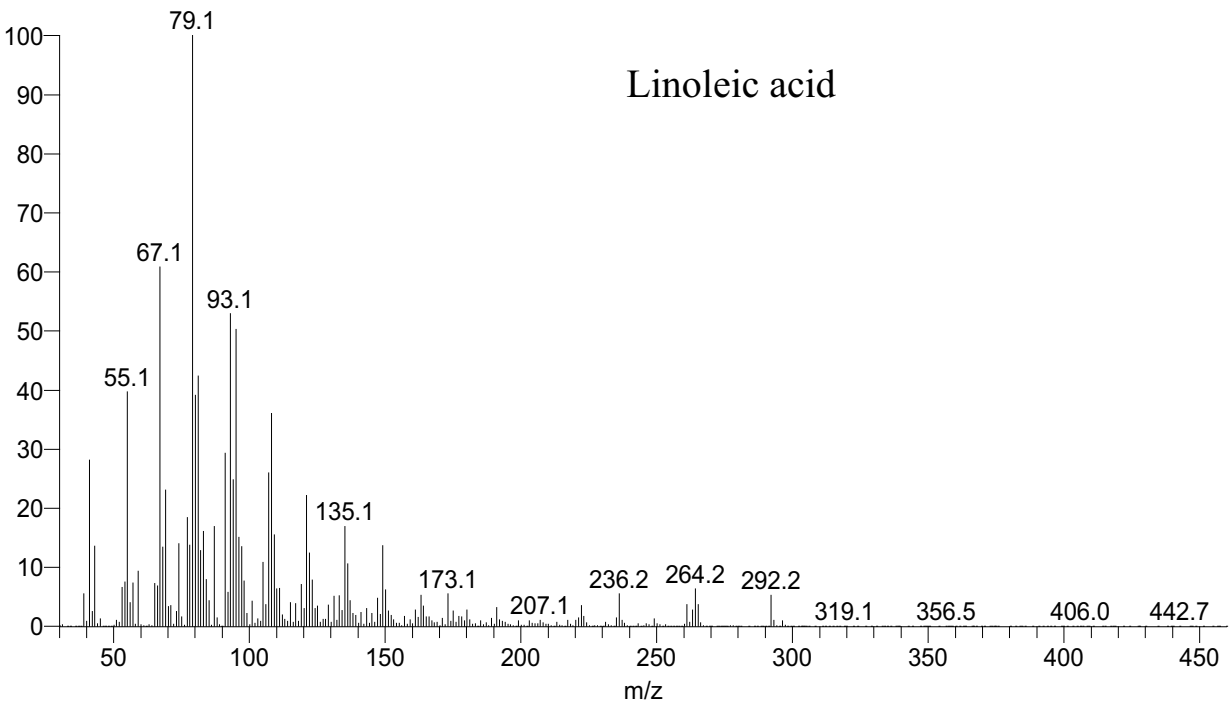

THREE4 \#3777 RT: 16.34 AV: 1 AV: 5 SB: 12 3770-3775 3779-3784 NL: 7.94E6

$\mathrm{T}:$ + c El Full ms [30.00-460.00]

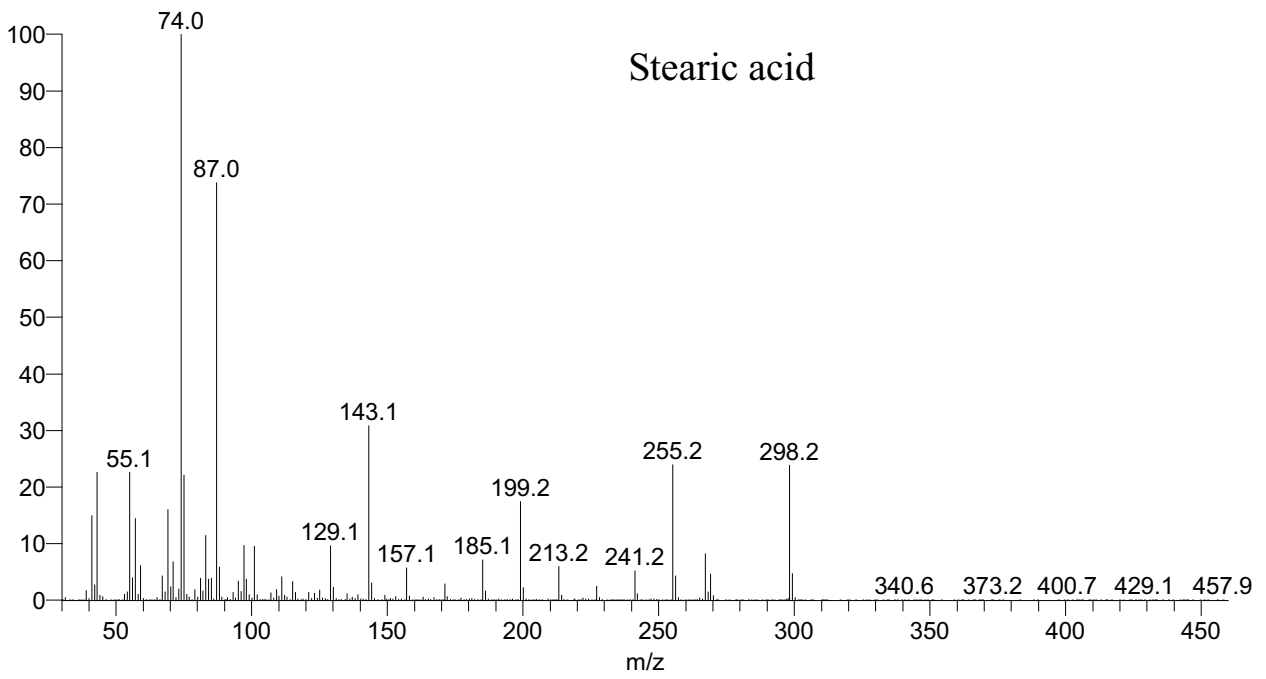

Supplemental Fig. 3. (Continued). 
FOUR3 \#693 RT: 5.85 AV: 1 AV: 5 SB: 12 686-691 695-700 NL: 1.36E7

$\mathrm{T}:+\mathrm{c}$ El Full ms [30.00-460.00]

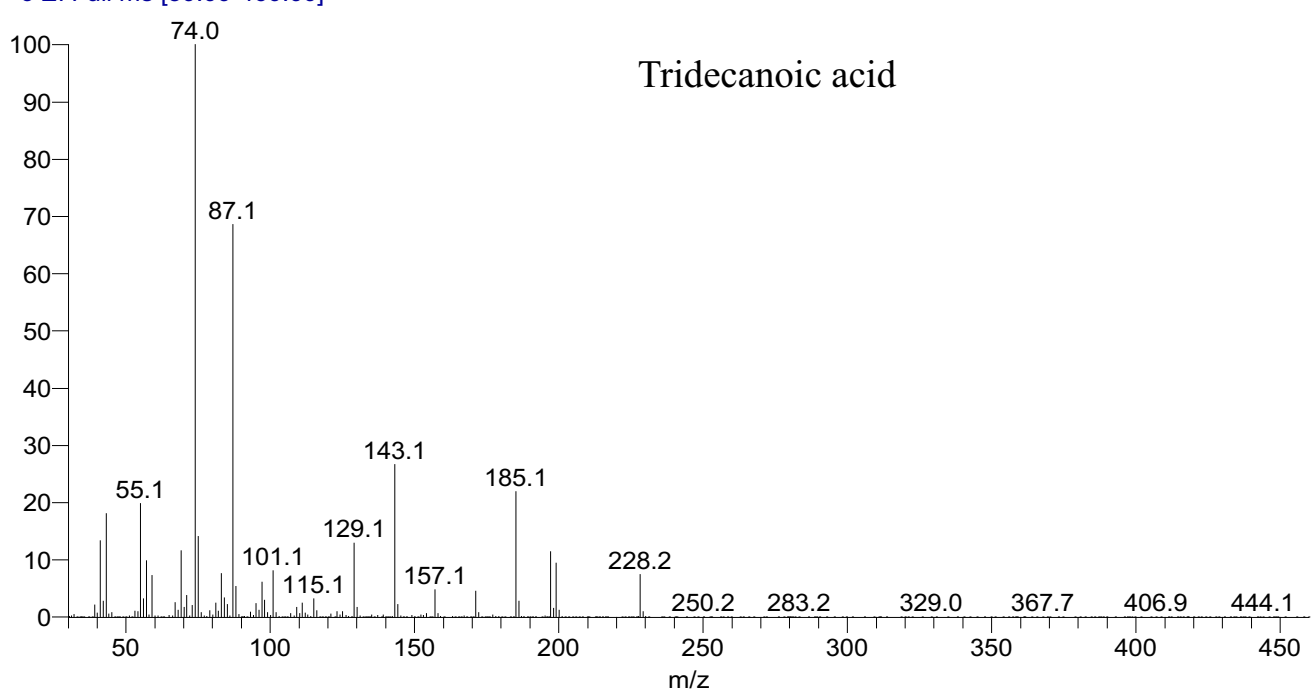

FOUR3 \#1430 RT: 8.36 AV: 1 AV: 5 SB: 12 1423-1428 1432-1437 NL: 5.12E4

T: + c El Full ms [30.00-460.00]

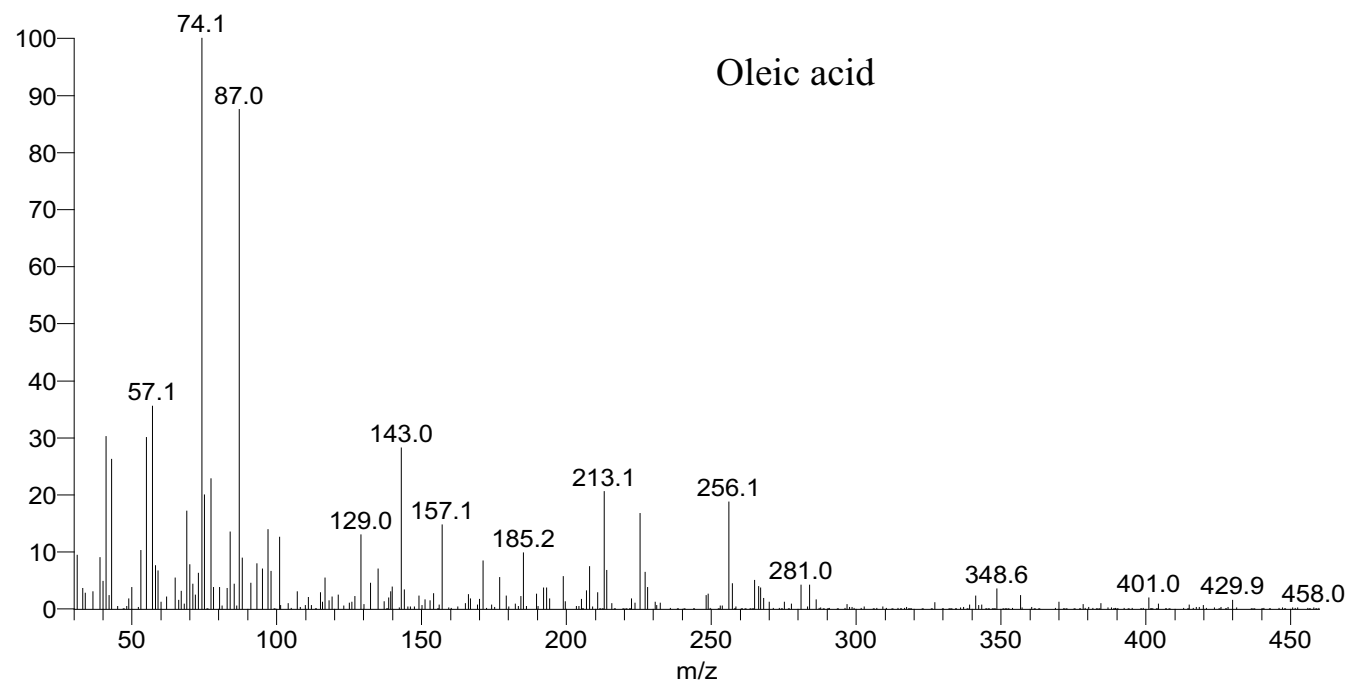

FOUR3 \#2010 RT: 10.33 AV: 1 AV: 5 SB: 12 2003-2008 2012-2017 NL: $4.82 E 7$ T: + c El Full ms [30.00-460.00]

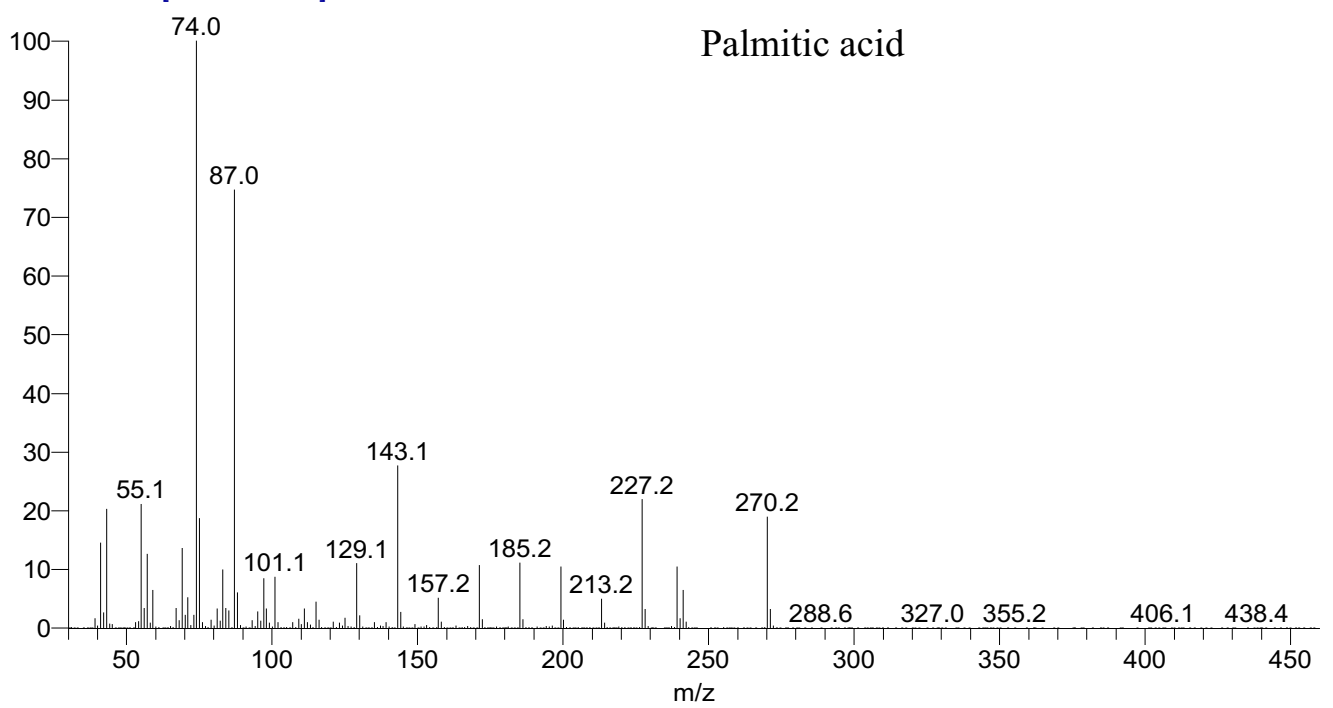

Supplemental Fig. 4. Ion map of six main fatty acids in Paeonia ostii seed oil with the understory intercropping cultivation method. 
FOUR3 \#3438 RT: 15.19 AV: 1 AV: 5 SB: 12 3431-3436 3440-3445 NL: 5.78E7

$\mathrm{T}:+\mathrm{c}$ El Full ms [30.00-460.00]

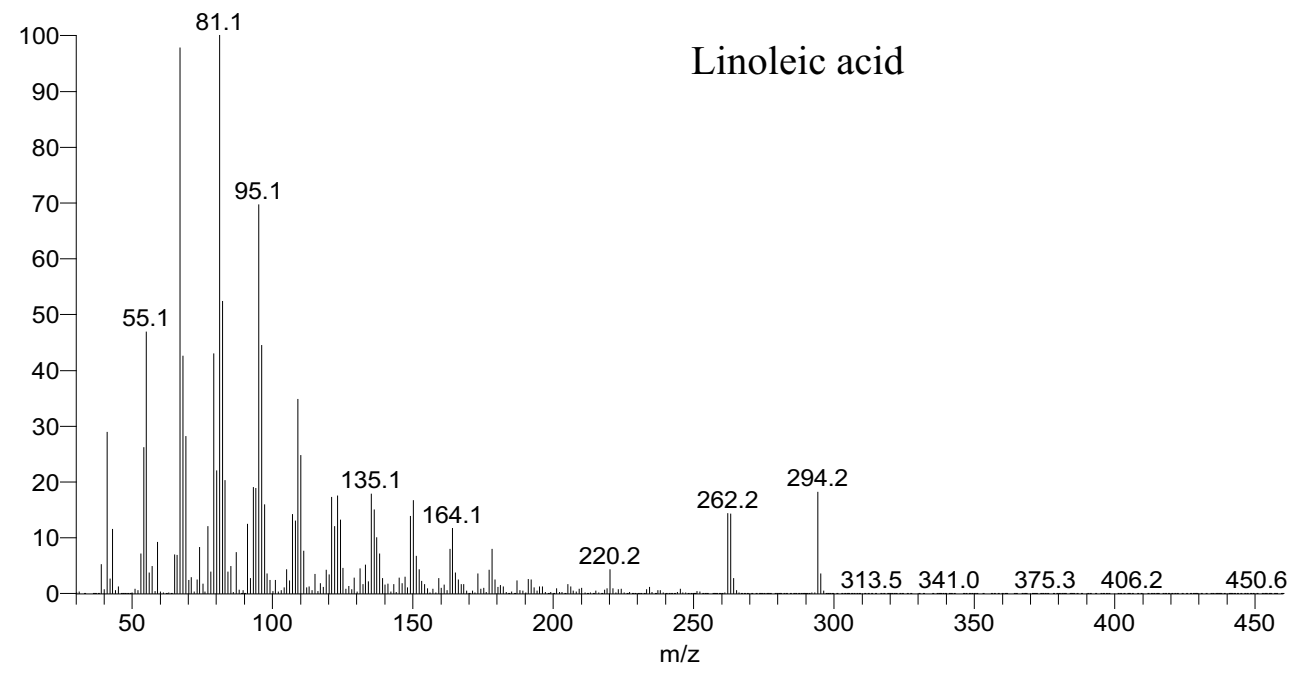

FOUR3 \#3522 RT: 15.48 AV: 1 AV: 5 SB: 12 3515-3520 3524-3529 NL: 1.09E8

T: + c El Full ms [30.00-460.00]

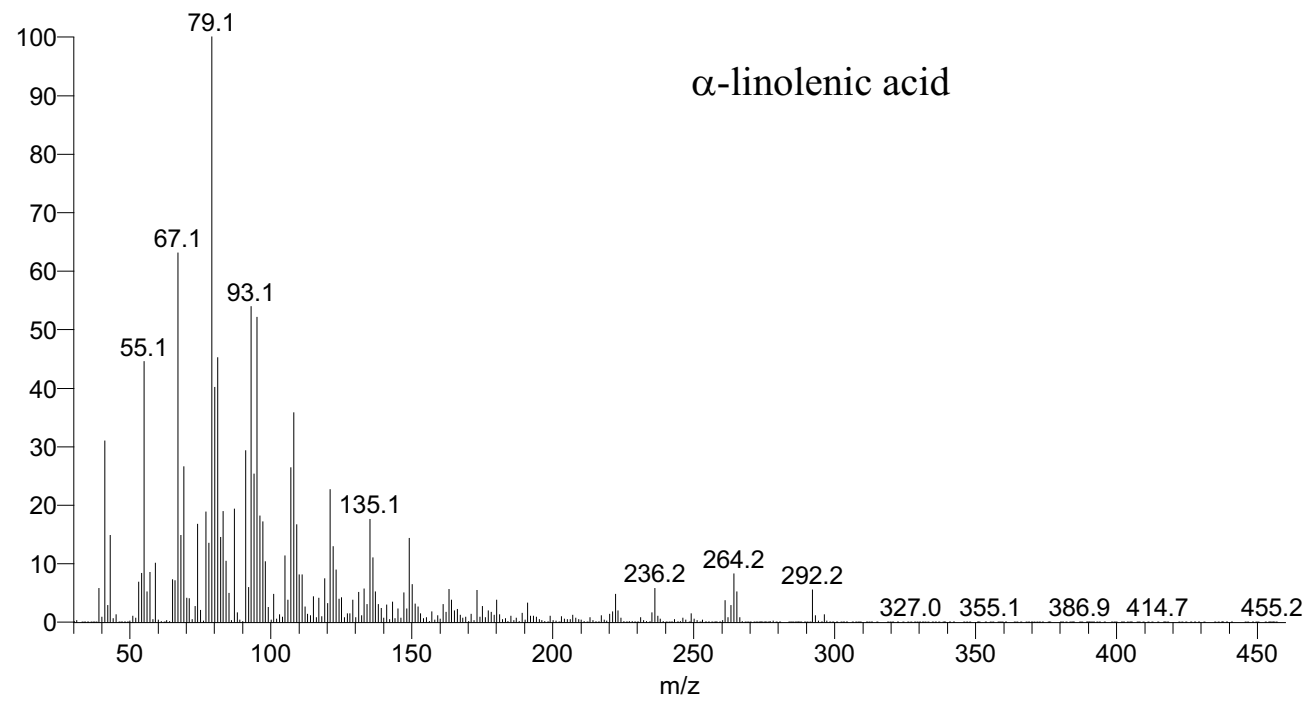

FOUR3 \#3776 RT: 16.34 AV: 1 AV: 5 SB: 12 3769-3774 3778-3783 NL: 8.01E6

$\mathrm{T}:+\mathrm{c}$ El Full ms [30.00-460.00]

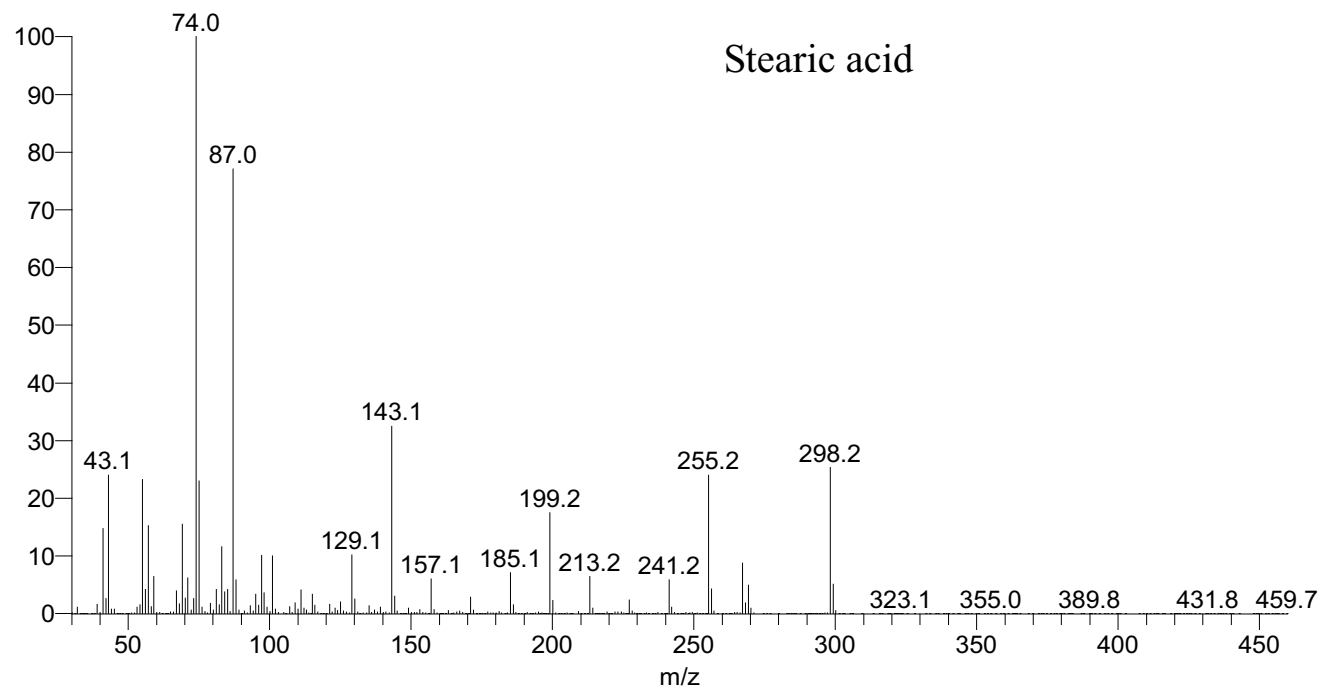

Supplemental Fig. 4. (Continued). 
Five3 \#694 RT: 5.86 AV: 1 AV: 5 SB: 12 687-692 696-701 NL: 1.67E7

$\mathrm{T}:$ + c El Full ms [30.00-460.00]

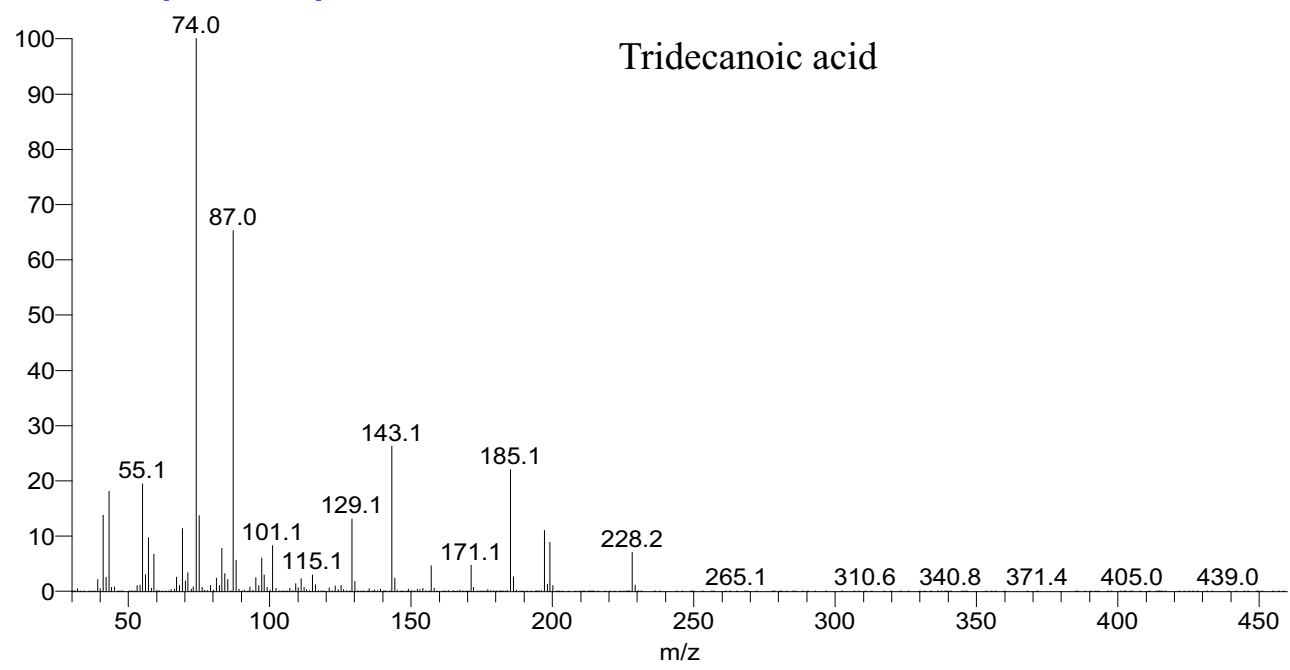

Five3 \#1430 RT: 8.36 AV: 1 AV: 5 SB: 12 1423-1428 1432-1437 NL: 9.48E4

$\mathrm{T}:$ + c El Full ms [30.00-460.00]

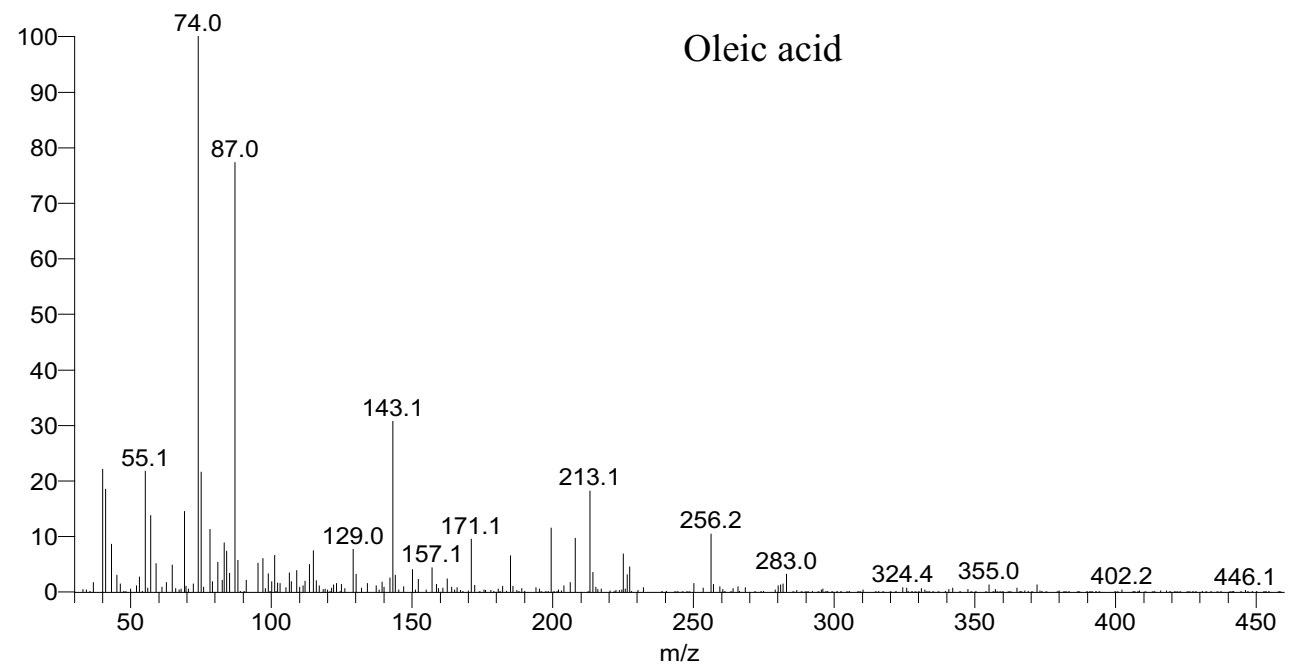

Five3 \#2011 RT: 10.34 AV: 1 AV: 5 SB: 12 2004-2009 2013-2018 NL: 4.89E7

T: + c El Full ms [30.00-460.00]

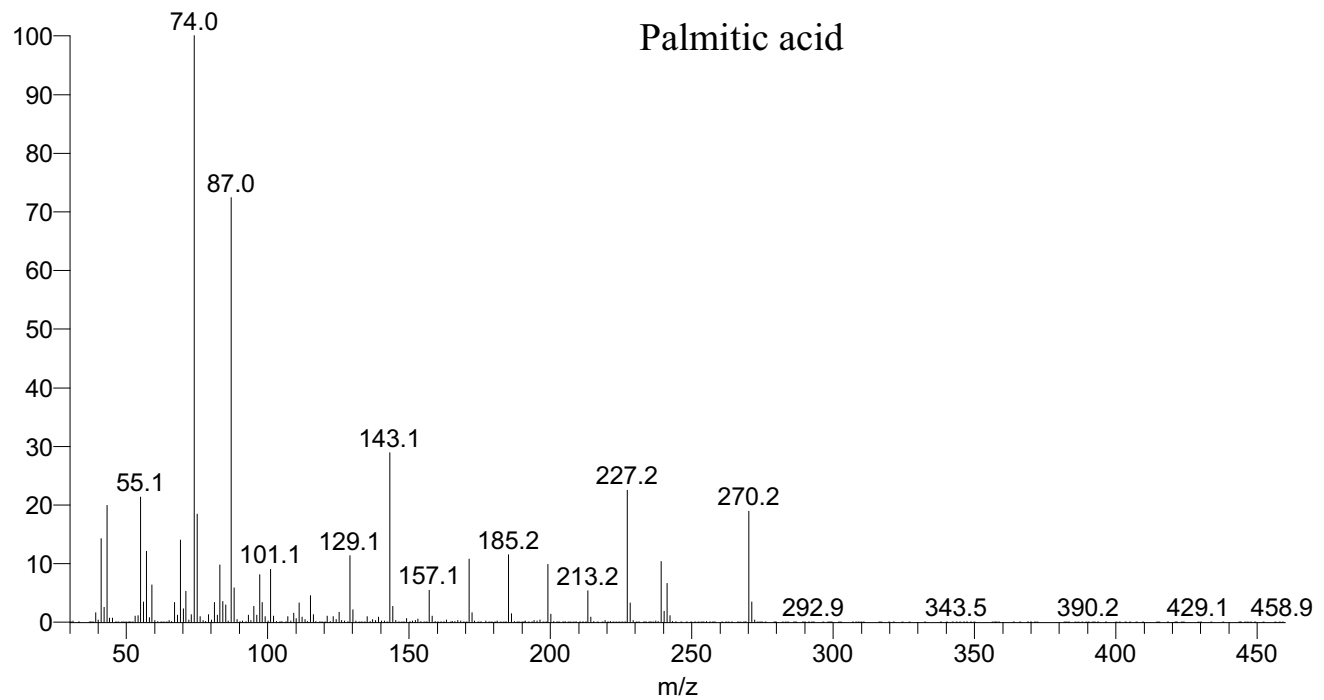

Supplemental Fig. 5. Ion map of six main fatty acids in Paeonia ostii seed oil with the open field cultivation method. 
Five3 \#3437 RT: 15.19 AV: 1 AV: 5 SB: 12 3430-3435 3439-3444 NL: 5.50E7

$\mathrm{T}:$ + c El Full ms [30.00-460.00]

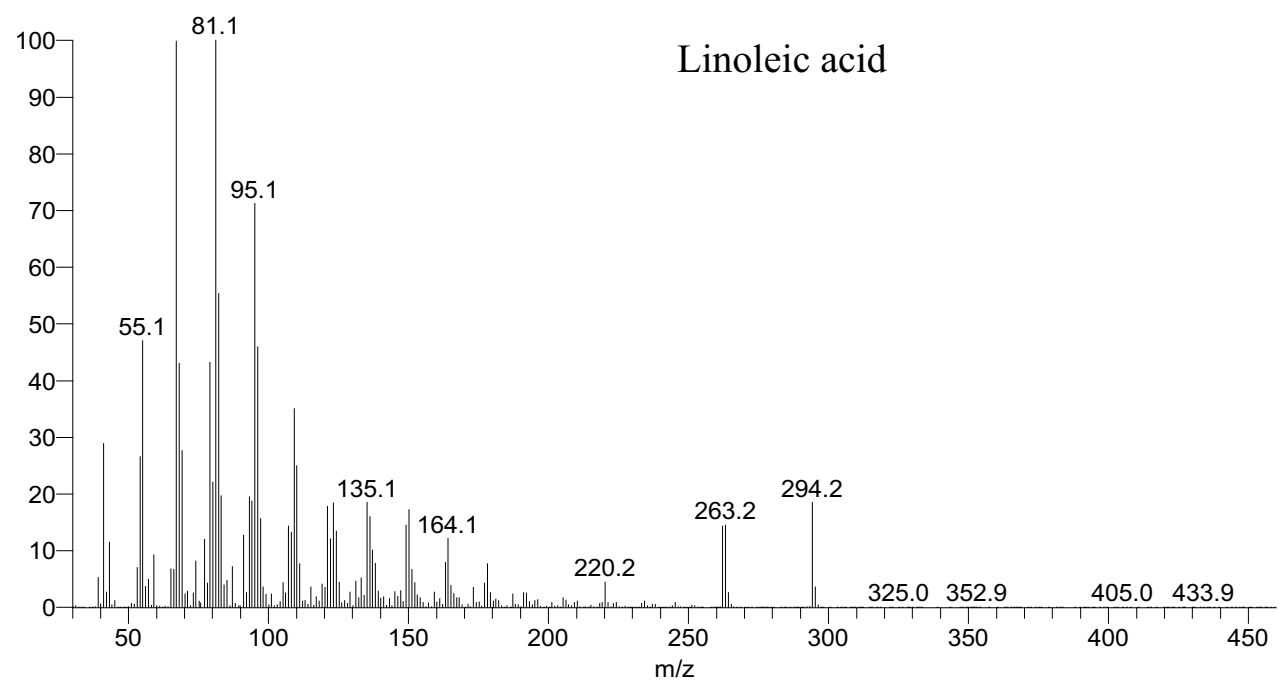

Five3 \#3523 RT: 15.48 AV: 1 AV: 5 SB: 12 3516-3521 3525-3530 NL: 1.07E8

$\mathrm{T}:$ + c El Full ms [30.00-460.00]

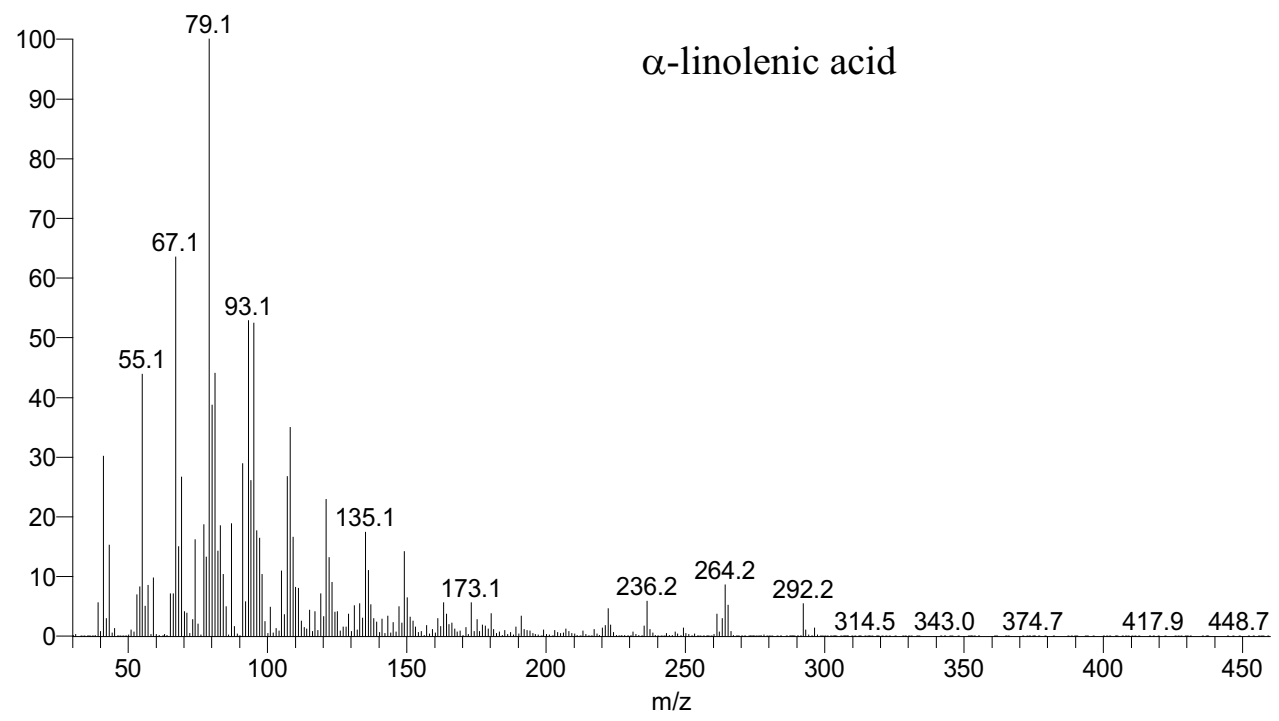

Five3 \#3777 RT: 16.34 AV: 1 AV: 5 SB: 12 3770-3775 3779-3784 NL: 6.95E6

$\mathrm{T}:$ + c El Full ms [30.00-460.00]

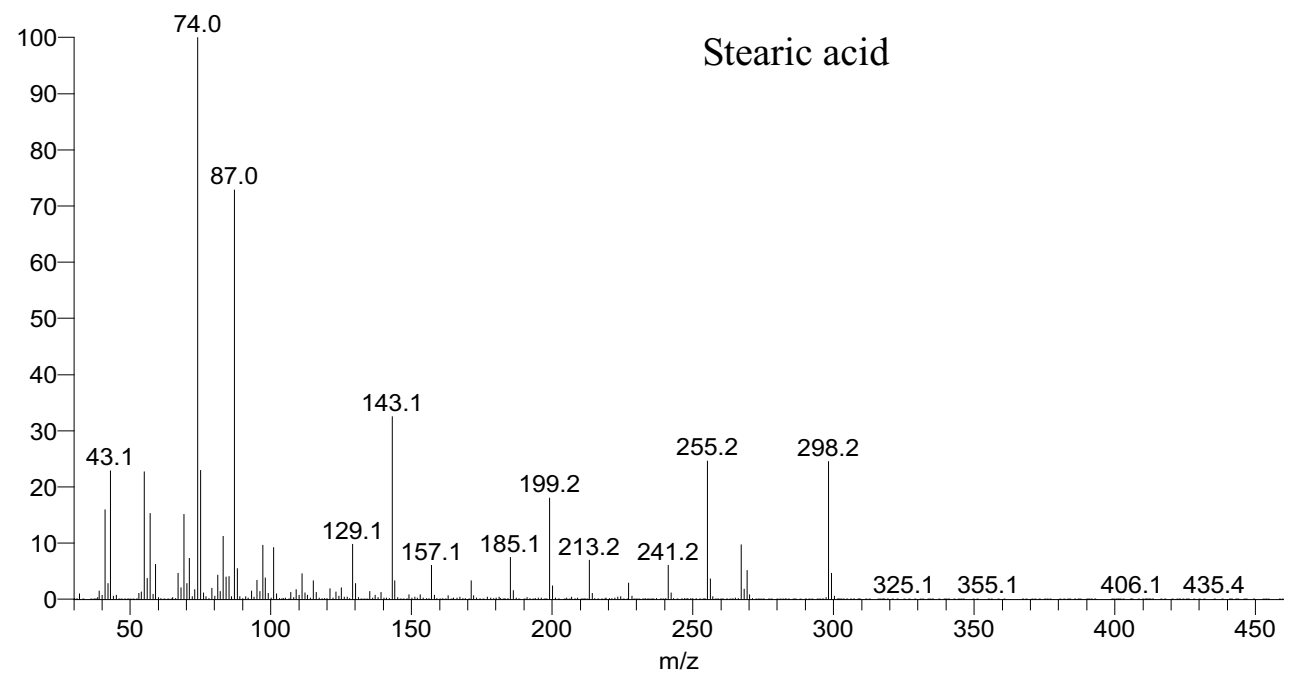

Supplemental Fig. 5. (Continued). 